\title{
Exploitative Competition of Microorganisms for Two Complementary Nutrients in Continuous Cultures
}

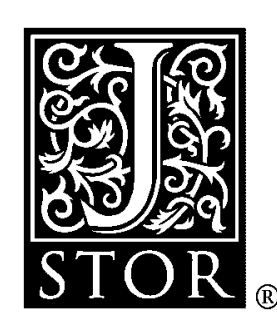

\author{
Sze-Bi Hsu; Kuo-Shung Cheng; S. P. Hubbell \\ SIAM Journal on Applied Mathematics, Vol. 41, No. 3. (Dec., 1981), pp. 422-444.
}

Stable URL:

http://links.jstor.org/sici?sici=0036-1399\%28198112\%2941\%3A3\%3C422\%3AECOMFT\%3E2.0.CO\%3B2-K

SIAM Journal on Applied Mathematics is currently published by Society for Industrial and Applied Mathematics.

Your use of the JSTOR archive indicates your acceptance of JSTOR's Terms and Conditions of Use, available at http://www.jstor.org/about/terms.html. JSTOR's Terms and Conditions of Use provides, in part, that unless you have obtained prior permission, you may not download an entire issue of a journal or multiple copies of articles, and you may use content in the JSTOR archive only for your personal, non-commercial use.

Please contact the publisher regarding any further use of this work. Publisher contact information may be obtained at http://www.jstor.org/journals/siam.html.

Each copy of any part of a JSTOR transmission must contain the same copyright notice that appears on the screen or printed page of such transmission.

The JSTOR Archive is a trusted digital repository providing for long-term preservation and access to leading academic journals and scholarly literature from around the world. The Archive is supported by libraries, scholarly societies, publishers, and foundations. It is an initiative of JSTOR, a not-for-profit organization with a mission to help the scholarly community take advantage of advances in technology. For more information regarding JSTOR, please contact support@jstor.org. 


\title{
EXPLOITATIVE COMPETITION OF MICROORGANISMS FOR TWO COMPLEMENTARY NUTRIENTS IN CONTINUOUS CULTURES*
}

\author{
SZE-BI HSU†, KUO-SHUNG CHENG $\dagger$ AND S. P. HUBBELL $\ddagger$
}

\begin{abstract}
This paper concerns the exploitative competition of two microorganisms for two complementary nutrients in the continuous culture. Consumption of the limiting resources follows the Holling Type II functional response or, equivalently, Michaelis-Menten kinetics, generalized to the two-resource situation. The predicted biological conditions which should give rise to each of the possible competitive outcomes are presented in detail and analyzed globally. A major conclusion is that each of the four outcomes of classical Lotka-Volterra two-species competition theory has multiple mechanistic origins in terms of consumerresource interactions. It is also shown that all four classical outcomes, including the case in which winning depends on the initial abundances of the competitors, can arise for this purely exploitative competition. Moreover, the outcomes of this exploitative competition can be predicted, in advance of actual competition, from measurements made on each species grown by itself on the resources.
\end{abstract}

1. Introduction. The classical theory of ecological competition between two or more species, attributed to Lotka [18] and Volterra [36], is an extension of the basic logistic model of single-species growth that dates from Verhulst [35]. The dynamical equations for this theory for two competitors, 1 and 2, are often written as

$$
\frac{d N_{1}}{d t}=r_{1} N_{1}\left\{\frac{1-\left(N_{1}+\alpha N_{2}\right)}{K_{1}}\right\}, \quad \frac{d N_{2}}{d t}=r_{2} N_{2}\left\{\frac{1-\left(\beta N_{1}+N_{2}\right)}{K_{2}}\right\},
$$

where $N_{i}$ is the number of the $i$ th competing species, $r_{i}$ and $K_{i}$ are the intrinsic rate of increase and the carrying capacity of the $i$ th competitor, respectively, and $\alpha$ and $\beta$ are the interaction of "competition" coefficients, expressing the per capita competitive effect of species 2 on 1 , and 1 on 2 , respectively. In the absence of competition $(\alpha=\beta=0)$, each population grows to its respective carrying capacity. In the presence of competition, one or the other rival may survive while its competitor dies out, or else. the rivals may coexist. These three biological outcomes result from the four mathematical cases that can occur provided that populations of both species are present initially. Competitive stability (coexistence) occurs when $\alpha<K_{1} / K_{2}$ and $\beta<K_{2} / K_{1}$; competitive instability (initial numbers of the competitors determine the eventual winner) occurs when these inequalities are both reversed; and competitive dominance (one or the other species wins regardless of initial numbers) occurs when one but not both of these inequalities are reversed.

The classical theory can perhaps be called a "phenomenological" theory insofar as it seeks to describe how the numbers of competing species change, and to predict the eventual outcome of such competition, without ever being specific about which limiting resources are the focus of competition, nor about how effectively the rival species forage for, and exploit, these resources. This classical theory has had an immense and lasting appeal because of its generality and simplicity, and it has been the subject of a very large number of theoretical studies (cf. Wangersky's review [38]). By the same token, however, this generality has also made it difficult for experimentalists to interpret and measure the theory's critical parameters. It has proven especially

* Received by the editors March 31, 1980, and in revised form December 1, 1980. This work was partially supported by the National Science Council of the Republic of China.

$\dagger$ Department of Applied Mathematics, National Chiao-Tung University, Hsin-Chu, Taiwan 300, Republic of China.

‡Department of Zoology, University of Iowa, Iowa City, Iowa 52240 . 
difficult to estimate the competition coefficients independently of actually growing the potential competitors together, and to determine their values under field conditions, although considerable attention has been devoted to these problems. Usually, competition coefficients have been estimated in laboratory competition studies by fitting the dynamical equations (1.1) to the growth curves of the species in competition (e.g., Vandermeer [34]). The attempts to estimate competition coefficients have usually focused on various measures of overlap in resource utilization (Schoener [28]), although information about whether the resources are limiting is usually lacking. Unfortunately, whenever these coefficients can only be estimated from the dynamics of populations already in competition, the value of the theory for prediction is thereby diminished. Thus, the classical theory has been attacked as a tautological exercise in curvefitting at best (Peters [23]). At worst the fit of (1.1) to data is poor (Wilbur [39], Neill [22], Richmond et al. [26]) because of a variety of nonlinearities in per capita rates of growth as a function of competitor densities.

Over the past 25 years the elements of a more mechanistic, resource-based theory of ecological competition have been under experimental and theoretical development by a diverse field of workers. A list of all significant contributions to this effort would be quite long, but some of the milestones have been papers by Herbert et al. [6], Powell [24], Holling [7], [8], [9], Miller [21], Dugdale [2], Eppley and Coatsworth [3], Epply and Thomas [4], Kilham [14], MacArthur [19], Stewart and Levin [30], Droop [1], Koch [15], Leon and Tumpson [17], Smith et al. [29], Taylor and Williams [31], Tilman and Kilham [32], Tilman [33], Real [25], and Hsu et al. [10], [12]. This theory considers the dynamics of the resources explicitly in addition to the population dynamics of the competing species. Moreover, it pays particular attention to the functional responses of the competitors to changes in resource density.

In comparison with the classical approach to competition, some things are gained and some are lost by adopting this approach. The disadvantages are that resource-based competition theory is usually less general, but also more difficult to analyze mathematically, than classical theory. However, the advantages are that the experimentalist has a specific set of somewhat more mechanistic questions to ask about limiting resources and the manner in which the consumers respond functionally and numerically to these resources. Thus, resource-based theory directs more explicit attention to resources and consumer-resource interactions than Lotka-Volterra theory which, classically at least, focused primarily if not exclusively on the phenomenological changes in competitor numbers. We believe that this approach will spur renewed interest in experimental studies of competition, and we also hope that it hastens the development of more predictive theory.

In this paper we present a resource-based competition model which describes how two microorganisms compete exploitatively for two complementary resources in the chemostat. A chemostat is a laboratory apparatus used for production and physiological study of microorganisms. In the chemostat model, the limiting nutrient is supplied at a constant rate. The input flow of medium contains all other factors for growth in excess. The output flow equals the input flow, and carries with it cells, waste product and unused nutrient. The model also approximates conditions for plankton growth in lakes, with the input of complementary limiting nutrients such as silica and phosphate from streams draining the surrounding watershed.

2. Competition for one resource. Before considering two resources, it is useful to review briefly what happens in the one-resource case. We assume that the consumption of a single limiting resource follows the Holling Type II functional response or, 
equivalently, Michaelis-Menten kinetics, which describes the chemical interaction between enzyme and substrate. When a species grows on a single limiting resource, there is some "break-even" concentration of that resource at which birth rate just balances death rate, which we will call the subsistence concentration. Now suppose that this limiting resource is supplied at a constant rate, corresponding to the constant carrying capacity environment of classical competition theory, and that two species (or more) are competing exploitatively for this resource. Then, provided that the resource is not being supplied at a rate below the subsistence concentration of all species, only a single species is predicted to survive: that species which has the smallest subsistence concentration of the resource (Stewart and Levin [30], Taylor and Williams [31], Hsu et al. [10]). Recently Hansen and Hubbell [5] conducted a rigorous test of this prediction. This nicely intuitive result turns out, however, to be dependent on having a constant resource input. If the resource has a periodic input, for example, seasonally (Stewart and Levin [30], Hsu [13]), or if the resource is a prey species capable of self-reproduction (Koch [15], Hsu et al. [12]), then there are conditions when the species can coexist in a dynamic, periodic fashion on a single resource (McGehee and Armstrong [20]). For the remainder of this paper, we will be concerned with the case of a constant carrying capacity environment, corresponding to Lotka-Volterra theory, but in resource terms.

The subsistence resource concentration is an important competition criterion, not only in one-resource situations, but in the two-resource theory discussed in this paper as well. For each species and limiting resource, there is such a concentration, potentially different in each case. It will be symbolized by $J$, following Rosenzweig [27].

In our previous work on this subject [10], [11], we have used $\lambda$ for this parameter, but henceforth we will use $J$ to avoid confusion with the finite rate of increase. The subsistence concentration can be calculated from three parameters that are measured on each species grown by itself on the particular resource. Thus, for resources $\boldsymbol{S}$ and species $i, J_{s i}$ can be found from: (a) the half-saturation constant for resource uptake, $K_{s i}$; (b) the intrinsic rate of increase, $r_{s i}$; and (c) the death rate, $D_{i}$. The half-saturation constant $K_{s i}$ corresponds to $K_{m}$ in Michaelis-Menten theory, and represents that concentration of resource at which consumption occurs at half the maximal rate. The subsistence concentration for species $i$ growth-limited by resource $S$ is the product of the half-saturation constant and the ratio of the death rate to the intrinsic rate of increase:

$$
J_{s i}=K_{s i}\left(\frac{D_{i}}{r_{s i}}\right) .
$$

The units of $J_{s i}$ are in resource concentration, since the units of $D_{i}$ and $r_{s i}$ cancel. The parameter $r_{s i}$ is equivalent to $r_{\max }$ for the $i$ th species. $r_{s i}=\left(m_{s i}-D_{i}\right)$, where $m_{s i}$ is the maximal per capita birth rate on resource $S$. For the detailed biological meaning of $J_{s i}$ we refer to [10].

The equations for single-resource competition among $n$ species for resource $S$ are

$$
\begin{gathered}
\frac{d S}{d t}=\left(S^{(0)}-S\right) D-\sum_{i=1}^{n} \frac{m_{s i}}{y_{s i}} \frac{S \cdot N_{i}}{K_{s i}+S}, \\
\frac{d N_{i}}{d t}=\frac{m_{s i} S \cdot N_{i}}{K_{s i}+S}-D_{i} N_{i}, \quad i=1,2, \cdots, n .
\end{gathered}
$$

$S$ is the concentration of resource, $S^{(0)}$ is the constant input concentration of resource, $D$ is the constant rate at which new nutrient is imported, as well as the rate at which 
nutrient at current concentration is exported. For the $i$ th organism, $N_{i}$ is the population density, $Y_{s i}$ is the yield of the $i$ th organism produced per unit of resource $S$ consumed, and $K_{s i}, m_{s i}$, and $D_{i}$ are as defined above. It will be recognized that $(2.2)$ is the system of equations for any continuous culture of several species, growth-limited by a single nutrient but supplied with all other required nutrients in nonlimiting amounts (Taylor and Williams [31], Hsu et al. [10], Hsu [11]). This is to be expected, since continuous cultures were specifically developed as idealized environments having a constant carrying capacity. Thus, it should be noted that a constant carrying capacity does not result from a fixed quantity of limiting resource, as in batch culture, but from a steady-state resource input-output situation. In (2.2), if no organisms are present, then the resource equilibrates at $S^{(0)}$ at the point when resource input and output rates are balanced.

The one-resource situation can be summed up as follows. With no loss of generality, number the competing species such that their $J$ 's are ordered, with $J_{s 1}<$ $J_{s 2}<\cdots<J_{s n}$. All species die out if the input concentration is less than the subsistence concentration for every species, i.e., if $S^{(0)}<J_{s 1}$. In this case, $\lim _{t \rightarrow \infty} S(t)=S^{(0)}$ and $\lim _{t \rightarrow \infty} N_{i}(t)=0, i=1, \cdots, n$. On the other hand, if $S^{(0)}>J_{s i}$ for any $i$, then species 1 survives and outcompetes all rival species. In this case, $\lim _{t \rightarrow \infty} S(t)=J_{s 1}, \lim _{t \rightarrow \infty} N_{1}(t)=$ $y_{s 1}\left(S^{(0)}-J_{s 1}\right)$ and $\lim _{t \rightarrow \infty} N_{i}(t)=0, i=2, \cdots, n$.

3. Competition for two resources. In situations involving two or more resources, it becomes necessary for the first time to consider how the resources, once consumed, interact to promote growth. Leon and Tumpson [17] have distinguished two important classes of resources: complementary and substitutable. Complementary resources are sources of different essential substances which are metabolically independent requirements for growth, such as a carbon source and a nitrogen source for a bacterium, or silica and phosphorus for a diatom. On the other hand, substitutable resources represent alternate sources of the same essential substance, and are metabolically interdependent requirements for growth, such as two carbon sources or two sources for phosphorous. In this paper we consider just the case of complementary resources.

Now consider two complementary resources, $R$ and $S$. For each consumer species there are two $J$ 's, one for each resource. These $J$ 's are the subsistence concentration of each resource when the species is growth-limited by that one resource alone. For species $i$, call these concentrations $J_{r i}$ and $J_{s i}$ of resources $R$ and $S$, respectively. These $J$ values determine the position of the zero-growth isocline for species $i$ on the $S-R$ resource plane.

The zero isocline for complementary resources is a pair of half-lines meeting at right angles at the point $\left(J_{s i}, J_{r i}\right)$ in the $S-R$ plane (Fig. 1). The lines are perpendicular because of the independence of the requirements for $R$ and $S$. In this case, growth is limited at any given time either by $R$ or by $S$, but not by both $R$ and $S$ simultaneously except at the corner). The curving dashed line passing through the corner in the isocline represents the equation, $m_{s i} S /\left(K_{s i}+S\right)=m_{r i} R /\left(K_{r i}+R\right)$, where the parameters are as previously defined but subscripted for the appropriate resource. Above the dashed line in the the $S$ - $R$ plane, species $i$ is $S$-limited, whereas below the dashed line, species $i$ is $R$-limited (Fig. 1). Thus, for example, when species $i$ is $S$-limited, no increase in resource $R$ in the region above the dashed curve will have any effect on increasing the growth rate of species $i$; only an increase in resource $S$ will have this effect. The converse is true in the region below the dashed curve.

Before presenting the competition models for two species on two complementary resources, we should discuss how the functional responses of the consumer species 


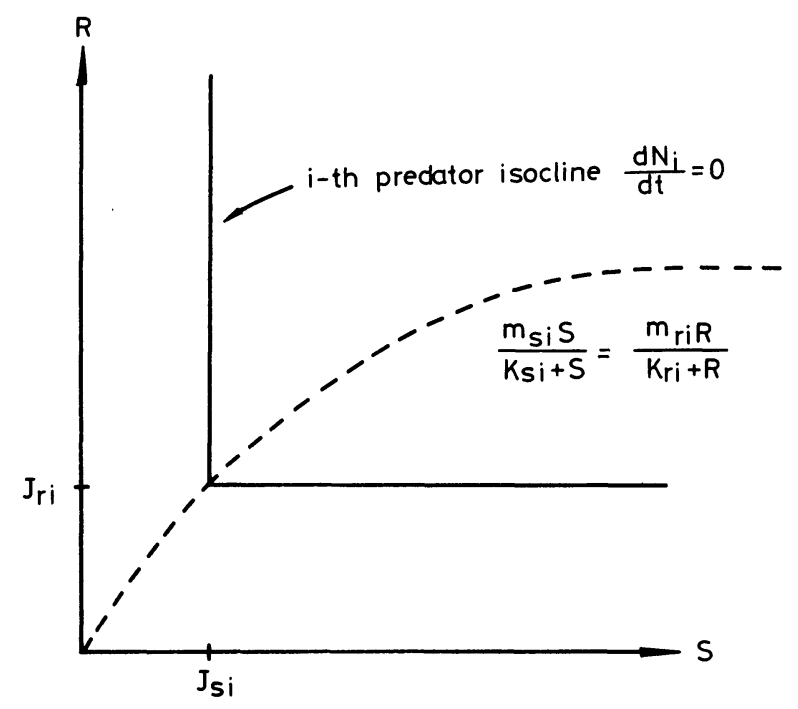

FIG. 1

have been generalized from one to two resources. In the one-resource case, the per capita consumption rate, according to the Type II functional response, is given by $\left(m_{r i} / y_{r i}\right)\left(R /\left(K_{r i}+R\right)\right)$ if the resource is $R$, or is given by $\left.\left(m_{s i} / y_{s i}\right)\left(S / K_{s i}+S\right)\right)$ if the resource is $S$. Now we generalize the functional response to two complementary resources. In this case, the per capita consumption rate of whichever resource is currently limiting growth is identical to the one-resource per capita consumption rate, as given above for the appropriate resource. The question then arises. At what rate is the nonlimiting resource consumed? This question can be answered when we consider the yield of consumer produced per unit of resource consumed. When the yield factors, $y_{r i}$ and $y_{s i}$ are constants, then it follows that there must be a fixed ratio of the growth-essential substances provided by resources $R$ and $S$ in a unit of consumer. Moreover, this also implies that the per capita consumption rate of the nonlimiting resource must be proportional to the per capita consumption rate of the limiting resource. If it were not, then the ratio of essential growth substances in the consumer would be changing, and the yield factors would no longer be constant. The proportionality constant is the ratio of the yield constants for the two resources. For example, suppose species $i$ is $S$-limited. Then the per capita consumption rate of $S$, call it $f_{1}(S)$, is

$$
f_{1}(S)=\frac{m_{s i}}{y_{s i}} \frac{S}{K_{s i}+S}
$$

whereas the concurrent per capita consumption rate of the nonlimiting resource $R$ is given by

$$
\frac{y_{s i}}{y_{r i}} \cdot f_{1}(S)=\frac{m_{s i}}{y_{r i}} \frac{S}{K_{s i}+S}
$$

Note that the expression in (3.2) does not contain the concentration of the nonlimiting resource $R$. Thus, it should be noted that: For complementary resources $R$ and $S$, when a species is $S$-limited, its per capita consumption rate of $R$ is independent of the concentration of $R$, whereas, when the species is $R$-limited, its per capita consumption rate of $S$ is independent of the concentration of $S$. The key to this statement is: "When 
is a species $S$-limited?". The species is only $S$-limited above a certain concentration of resource $R$ (above the dashed line in Fig. 1). Below this concentration of $R$, the per capita consumption rate of $R$ does depend on the concentration of $R$; but this dependence is because the species is now $R$-limited and no longer $S$-limited. The converse argument applies when the species is $R$-limited.

4. Statement of the model. Given the preceding development of the biological basis for the functional response of species exploiting pairs of complementary resources, it is a straightforward matter to state the two-resource, two-species competition model in the continuous culture.

For complementary resources, $R$ and $S$, and species 1 and 2 competing exploitatively for them, the system of equations is

$$
\begin{gathered}
\frac{d S}{d t}=\left(S^{(0)}-S\right) D-\frac{1}{y_{s 1}} g_{1}(S, R) N_{1}-\frac{1}{y_{s 2}} g_{2}(S, R) N_{2}, \\
\frac{d R}{d t}=\left(R^{(0)}-R\right) D-\frac{1}{y_{r 1}} g_{1}(S, R) N_{1}-\frac{1}{y_{r 2}} g_{2}(S, R) N_{2}, \\
\frac{d N_{1}}{d t}=\left[g_{1}(S, R)-D\right] N_{1}, \quad \frac{d N_{2}}{d t}=\left[g_{2}(S, R)-D\right] N_{2}, \\
S(0)>0, \quad R(0)>0, \quad N_{1}(0)>0, \quad N_{2}(0)>0,
\end{gathered}
$$

where

$$
\begin{aligned}
& g_{1}(S, R)=\min \left(\frac{m_{s 1} S}{K_{s 1}+S}, \frac{m_{r 1} R}{K_{r 1}+R}\right), \\
& g_{2}(S, R)=\min \left(\frac{m_{s 2} S}{K_{s 2}+S}, \frac{m_{r 2} R}{K_{r 2}+R}\right) .
\end{aligned}
$$

As noted previously, this model assumes constant carrying capacities (fixed rate of nutrient input), constant yield factors (unit of consumer produced per unit of resource consumed), and Type II functional responses, generalized to two complementary resources. In addition, we have assumed Type II numerical responses in per capita birth rates, with a direct dependence on the external supply of resources. Finally, we have assumed the same dilution rate $D$ for $S, R, N_{1}, N_{2}$. The parameters in (4.11) have all been previously defined at various points in the text, but it is convenient to relist them here in one place for ease of reference:

$S^{(0)}, R^{(0)}=$ input concentrations of resource $S$ and $R$, respectively.

$D=$ input flow rate of medium containing $S, R$ and also the output flow rate of medium containing unused $S, R$ and cells $N_{1}, N_{2}$.

$m_{s i}, m_{r i}=$ maximal per capita birth rate of species $i$ on resource $S$ or $R$ alone.

$y_{s i}, y_{r i}=$ yield of species $i$ per unit of resource $S$ or $R$ consumed.

$K_{s i}, K_{r i}=$ half-saturation constant for species $i$ on resource $S$ or $R$.

We analyze the behavior of solutions of this system of ordinary differential equations in order to answer the biological question, under what conditions will neither, one, or both species survive or die out? We also seek to determine the limiting behavior of the surviving species and the resources.

5. Statement of results. In this section we state the principal results of the paper. The proofs and certain technical lemmas are deferred to $\S 6$. The first lemma is a statement that the system given by (4.1) is as "well-behaved" as one intuits from the 
biological problem. The proof of the lemma is similar to that in [10], and we omit it.

LEMMA 5.1. Solutions of (4.1) are positive and bounded. Furthermore, we have

$$
\begin{aligned}
& S(t)=S^{(0)}-\sum_{i=1}^{2} \frac{N_{i}(t)}{y_{s i}}+o(1) \quad \text { as } t \rightarrow \infty \\
& R(t)=R^{(0)}-\sum_{i=1}^{2} \frac{N_{i}(t)}{y_{r i}}+o(1) \quad \text { as } t \rightarrow \infty
\end{aligned}
$$

The next lemma provides conditions under which the organisms cannot survive given the fixed dilution rate and the fixed input rates of the nutrients. Before we state the lemma, we note the following two pairs of equivalent statements, namely,

$$
\begin{aligned}
& \frac{m_{s i} S^{(0)}}{K_{s i}+S^{(0)}}<D \quad \text { if and only if } \quad m_{s i} \leqq D \text { or } \frac{K_{s i} D}{m_{s i}-D}>S^{(0)} \\
& \frac{m_{r i} R^{(0)}}{K_{r i}+R^{(0)}}<D \quad \text { if and only if } \quad m_{r i} \leqq D \text { or } \frac{K_{r i} D}{m_{r i}-D}>R^{(0)}
\end{aligned}
$$

LEMMA 5.2. If

$$
\frac{m_{s i} S^{(0)}}{K_{s i}+S^{(0)}}<D \quad \text { or } \quad \frac{m_{r i} R^{(0)}}{K_{r i}+R^{(0)}}<D
$$

then $\lim _{t \rightarrow \infty} N_{i}(t)=0$.

Lemma 5.2 states the necessary conditions for species $N_{i}$ to survive, i.e.,

$$
0<\frac{K_{s i} D}{m_{s i}-D}<S^{(0)} \text { and } 0<\frac{K_{r i} D}{m_{r i}-D}<R^{(0)} .
$$

Since nutrients $S$ and $R$ are complementarily essential to the growth of species $N_{i}$, there are minimum input concentrations $S^{(0)}$ and $R^{(0)}$ both for $S$ and $R$ in order to support the species $N_{i}$.

COROllaRY 5.3. If (5.3) holds for $i=1,2$, then $\lim _{t \rightarrow \infty} S(t)=S^{(0)}, \lim _{t \rightarrow \infty} R(t)=$ $R^{(0)}$ and $\lim _{t \rightarrow \infty} N_{i}(t)=0, i=1,2$.

We state the principal result in the case of inadequate input concentration of nutrients in four parts. We are able to determine the globally asymptotic behavior of the solutions in Theorem 5.4. The theorem may be summarized by noting that the unsuccessful competitor does not affect the eventual behavior of the survivor and its resources.

Before we state Theorem 5.4, we introduce the following important parameters:

$$
\begin{gathered}
J_{s i}=\frac{K_{s i} D}{m_{s i}-D}, \quad J_{r i}=\frac{K_{r i} D}{m_{r i}-D}, \quad i=1,2, \\
C_{i}=\frac{y_{s i}}{y_{r i}}, \quad T_{i}=\frac{R^{(0)}-J_{r i}}{S^{(0)}-J_{s i}}, \quad i=1,2 .
\end{gathered}
$$

THEOREM 5.4(i), (ii). Let (5.3) hold for $i=2$ and $0<J_{s 1}<S^{(0)}, 0<J_{r 1}<R^{(0)}$.

(i) If $T_{1}<C_{1}$, then the trajectory of (4.1) approaches the equilibrium $\left(E_{s 1}\right)$ as $t \rightarrow \infty$, where

$$
\left(E_{s 1}\right)=\left(J_{s 1}, R_{s 1}^{*}, N_{s 1}^{*}, 0\right), \quad N_{s 1}^{*}=y_{s 1}\left(S^{(0)}-J_{s 1}\right), \quad R_{s 1}^{*}=R^{(0)}-\frac{N_{s 1}^{*}}{y_{r 1}} .
$$


(ii) If $T_{1}<C_{1}$, then the trajectory of (4.1) approaches the equilibrium $\left(E_{r 1}\right)$ as $t \rightarrow \infty$, where

$$
\left(E_{r 1}\right)=\left(S_{r 1}^{*}, J_{r 1}, N_{r 1}^{*}, 0\right), \quad N_{r 1}^{*}=y_{r 1}\left(R^{(0)}-J_{r 1}\right), \quad S_{r 1}^{*}=S^{(0)}-\frac{N_{r 1}^{*}}{y_{s 1}} .
$$

THEOREM 5.4(iii), (iv). Let (5.3) hold for $i=1$ and $0<J_{s 2}<S^{(0)}, 0<J_{r 2}<R^{(0)}$. where

(iii) If $T_{2}>C_{2}$, then the trajectory of (4.1) approaches the equilibrium $\left(E_{s 2}\right)$ as $t \rightarrow \infty$,

$$
\left(E_{s 2}\right)=\left(J_{s 2}, R_{s 2}^{*}, 0, N_{s 2}^{*}\right), \quad N_{s 2}^{*}=y_{s 2}\left(S^{(0)}-J_{s 2}\right), \quad R_{s 2}^{*}=R^{(0)}-\frac{N_{s 2}^{*}}{y_{r 2}} .
$$
where

(iv) If $T_{2}<C_{2}$, then the trajectory of (4.1) approaches the equilibrium $\left(E_{r 2}\right)$ as $t \rightarrow \infty$,

$$
\left(E_{r 2}\right)=\left(S_{r 2}^{*}, J_{r 2}, 0, N_{r 2}^{*}\right), \quad N_{r 2}^{*}=y_{r 2}\left(R^{(0)}-J_{r 2}\right), \quad S_{r 2}^{*}=S^{(0)}-\frac{N_{r 2}^{*}}{y_{s 2}} .
$$

Theorem 5.4 states that when $\lim _{t \rightarrow \infty} N_{i}(t)=0$ the equation (4.1) is reduced to a system of three ordinary differential equations. In order to present the biological meaning of parameters $T_{i}, C_{i}$, we assume $\lim _{t \rightarrow \infty} N_{2}(t)=0$. We may rewrite $T_{1}, C_{1}$ as

$$
T_{1}=\frac{\left(R^{(0)}-J_{r 1}\right) D}{\left(S^{(0)}-J_{s 1}\right) D}, \quad C_{1}=\frac{1 / y_{r 1}}{1 / y_{s 1}} .
$$

When only species 1 is present, $T_{1}$ represents the ratio of the steady-state nutrient regeneration rates at equilibrium under consumption by $1 . J_{r 1}$ and $J_{s 1}$ are the equilibrium concentrations of resources $R$ and $S$, respectively, under steady-state consumption by species 1 . The parameter $C_{1}$ represents the fixed yield ratio for species 1 growing on resources $R$ and $S$. The units of $\left(1 / y_{r 1}\right)$ are (units $R$ consumed/unit species 1 produced); thus $C_{1}$ is the ratio, (units $R$ consumed/units $S$ consumed) per unit of species 1 produced.

By comparing $T_{1}$ with $C_{1}$, we can determine whether species 1 is $S$-limited or $R$-limited. This is because $C_{1}$ represents the invariant ratio in which the essential nutrients $R$ and $S$ are consumed by species 1 , whereas $T_{1}$ represents the ratio in which these same resources are being externally regenerated under steady-state consumption pressure from species 1 . Therefore, if $T_{1}>C_{1}$, the growth rate of species 1 is $S$-limited because $S$ is regenerating at a steady-state rate slower than $R$ with respect to the required consumption ratio for species 1 . Similarly, if $T_{1}<C_{1}$, the growth rate of species 1 will be $R$-limited. The above considerations also apply to species 2 for appropriate values of $T_{2}$ and $C_{2}$.

Definition. If $T_{1}>C_{1}$ (or $T_{1}<C_{1}$ ), we say that species $N_{1}$ is $S$-limited (or $R$-limited). Similarly, if $T_{2}>C_{2}$ (or $T_{2}<C_{2}$ ), we say that species $N_{2}$ is $S$-limited (or $R$-limited).

Remark. We may justify the concept of $R$-limited or $S$-limited. Consider system (4.1) with $N_{2} \equiv 0$. Suppose $N_{1}$ is $R$-limited, then at equilibrium $\left(S_{r 1}^{*}, J_{r 1}, N_{r 1}^{*}\right)$ we have $D=\left(m_{r 1} J_{r 1}\right) /\left(K_{r 1}+J_{r 1}\right)<\left(m_{s 1} S_{r 1}^{*}\right) /\left(K_{s 1}+S_{r 1}^{*}\right)$ and hence $S_{r 1}^{*}>J_{s 1}$. Then

$$
y_{r 1}\left(R^{(0)}-J_{r 1}\right)=\frac{m_{r 1} J_{r 1}}{K_{r 1}+J_{r 1}}, \quad \frac{N_{r 1}^{*}}{D}=y_{s 1}\left(S^{(0)}-S_{r 1}^{*}\right)<y_{s 1}\left(S^{(0)}-J_{s 1}\right)
$$

and so $T_{1}<C_{1}$. Similarly, if $N_{1}$ is $S$-limited then $C_{1}<T_{1}$. 
In order to discuss the interior equilibrium point, we may assume as a basic hypothesis

$$
S^{(0)}>J_{s 1}, \quad J_{s 2}>0, \quad R^{(0)}>J_{r 1}, \quad J_{r 2}>0 .
$$

Under the assumption (H1), the equations of (4.1) may be relabeled without loss of generality, so that we may assume either

$$
J_{r 1}<J_{r 2}, \quad J_{s 1}<J_{s 2}
$$

or

$$
J_{r 1}<J_{r 2}, \quad J_{s 2}<J_{s 1} .
$$

We note that most of the conditions on parameters for the various cases in Theorems, 5.5, 5.6 can also be established by the linearization method.

Before we state our main results, Theorems 5.5 and 5.6, we introduce the following parameters:

$$
T^{*}=\frac{R^{(0)}-J_{r 2}}{S^{(0)}-J_{s 1}}, \quad N_{1 c}^{*}=\frac{y_{s 1}\left(S^{(0)}-J_{s 1}\right)\left(C_{2}-T^{*}\right)}{C_{1}-C_{1}}, \quad N_{2 c}^{*}=\frac{y_{s 2}\left(S^{(0)}-J_{s 1}\right)\left(T^{*}-C_{1}\right)}{C_{2}-C_{1}}
$$

Theorem 5.5. Assume (H1) and (H2) hold (see Figs. 2a, 2b). Let $C_{1} \neq C_{2}$. Then the statements in Theorem 5.4(i) (ii) hold.

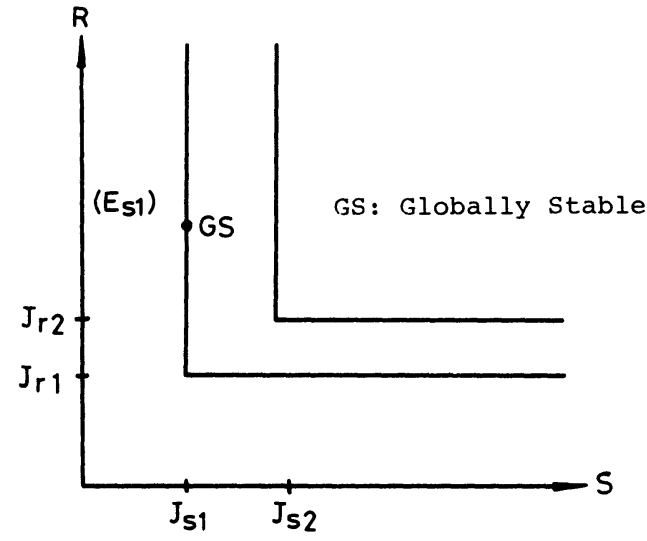

a. $\quad N_{1}$ is $S$-limited.

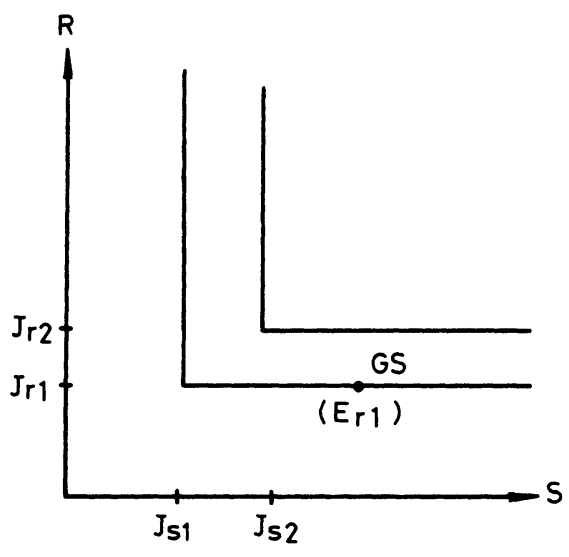

b. $\boldsymbol{N}_{1}$ is $R$-limited.

FiG. 2

Theorem 5.5 states that, if one species has the lower $J$ 's for both nutrients $S$ and $R$, then that species will survive and its rival will not.

Now we consider the situation when each species has the lower subsistence concentration on one of the resources, say, $J_{r 1}<J_{r 2}, J_{s 2}<J_{s 1}$. Then the parameters $T^{*}$, $C_{1}$ and $C_{2}$ become important in the competition outcomes. other.

First we state the following results describing how one species can outcompete the

Theorem 5.6(i). Assume (H1) and (H3) hold. Let $C_{1} \neq C_{2}$. If $T^{*}<C_{1}, C_{2}$, then the statements in Theorem 5.4(i), (ii) hold (see Figs. 3a, 3b).

THEOREM 5.6(ii). Under the assumptions of part (i), if $T^{*}>C_{1}, C_{2}$, then the statements in Theorem 5.4(iii), (iv) hold (see Figs. 4a, 4b). 

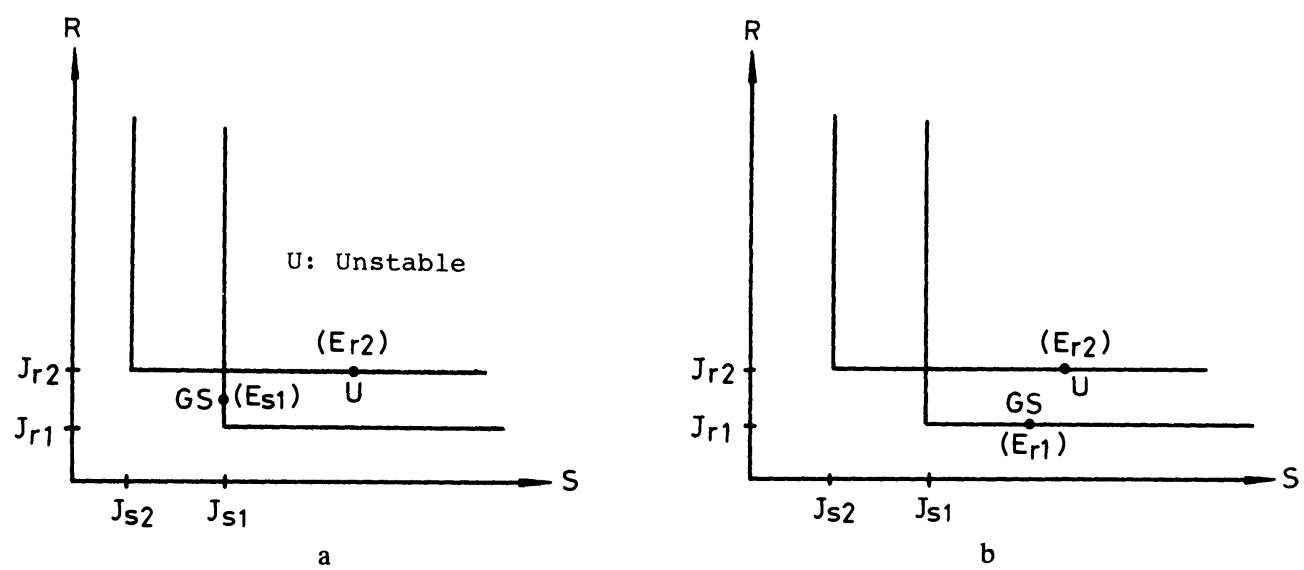

FIG. 3
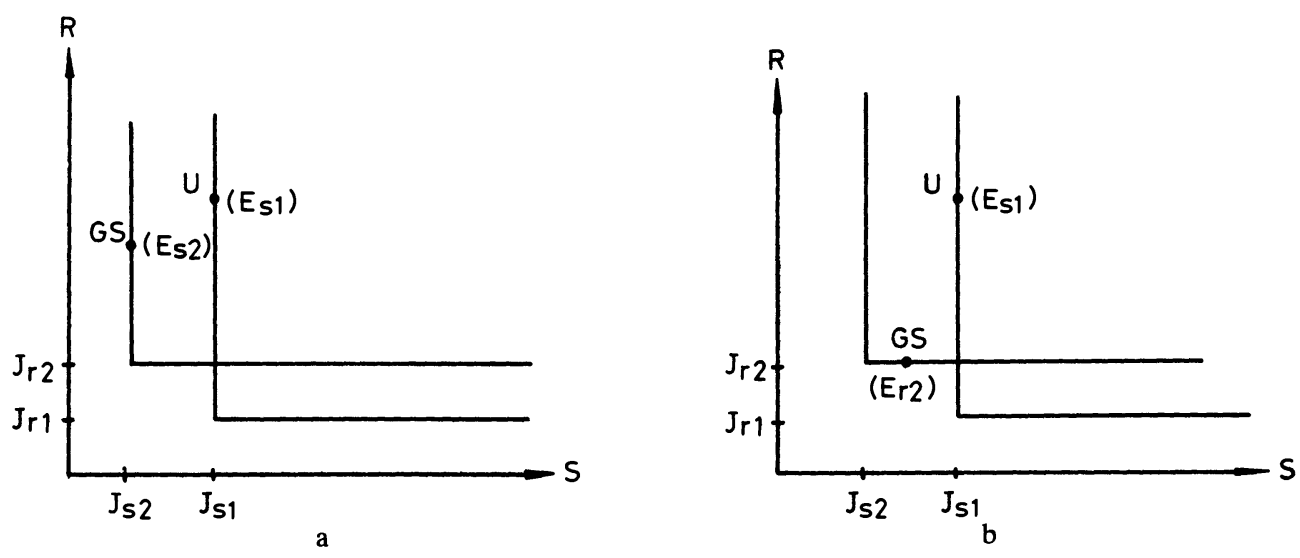

FIG. 4

In order to explain the biological meaning of Theorem 5.6(i), (ii), we rewrite $T^{*}=\left(R^{(0)}-J_{r 2}\right) D /\left(S^{(0)}-J_{s 1}\right) D$, which represents the ratio of the steady-state regeneration rate of $R$ when $N_{2}$ is alone and that of $S$ when $N_{1}$ is alone. We note that under the assumption (H3) we have $T^{*}<T_{1}$ and $T^{*}>T_{2}$. First we consider Theorem 5.6(i). The assumption $T^{*}<C_{1}, C_{2}$ implies $T_{2}<C_{2}$; i.e., species $N_{2}$ is always $R$-limited. But, since species $N_{1}$ has the lower subsistence concentration for resource $R$, species $N_{1}$ always wins. Note, however, that at the one-species equilibrium, species $N_{1}$ may again be either $S$-limited or $R$-limited. Similarily, we can explain Theorem 5.6(ii). Species $N_{2}$ outcompetes species $N_{1}$ for the reason that species $N_{1}$ is $S$-limited and $N_{2}$ has the lower subsistence concentration for resource $S$.

Secondly, we describe how two species can coexist.

THEOREM 5.6(iii). Under the assumptions of part (i). If $C_{1}<T^{*}<C_{2}$, then the "positive" equilibrium $\left(E_{c}\right)=\left(J_{s 1}, J_{r 2}, N_{1 c}^{*}, N_{2 c}^{*}\right)$ exists and is globally asymptotically stable in the first orthant (see Fig. 5).

In this case, we note that $C_{1}<T_{1}$, and $T_{2}<C_{2}$; i.e., $N_{1}$ is $S$-limited and $N_{2}$ is $R$-limited. But, species $N_{1}, N_{2}$ has lower subsistence concentration for $R$ and $S$ respectively. Coexistence occurs because each species has the lower subsistence concentration for that resource which, at the two-species equilibrium mixture of resources, most limits the growth of its rival. 


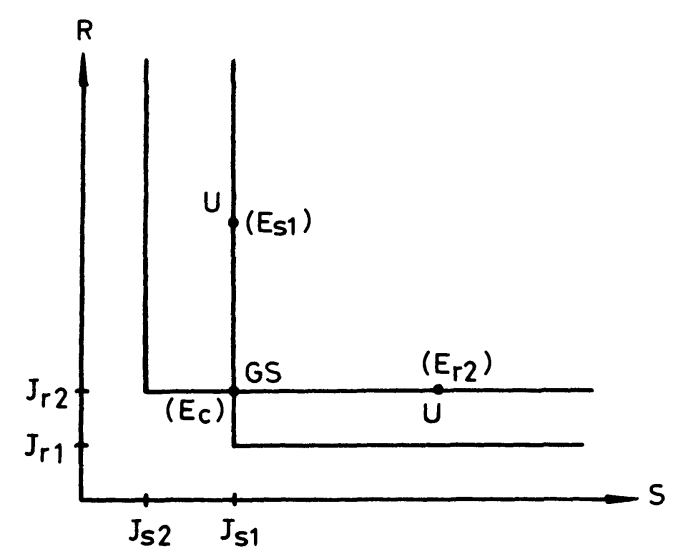

FIG. 5

Finally, we state a result describing the outcome of competition depends on initial populations. There are two cases. The first one $((a),(d))$ is the case when both species are limited by the same resource. The second one $((b),(d))$ is the case when the species are limited by different resources.

THEOREM 5.6(iv). Under the assumptions of part (i), if $C_{2}<T^{*}<C_{1}$, then $\left(E_{c}\right)$ exists and unstable. Furthermore, we have four possible outcomes:

(a) If $N_{1}$ is $S$-limited and $N_{2}$ is $S$-limited, then $\left(E_{s 1}\right)$ and $\left(E_{s 2}\right)$ are asymptotically stable. (see Fig. 6a).
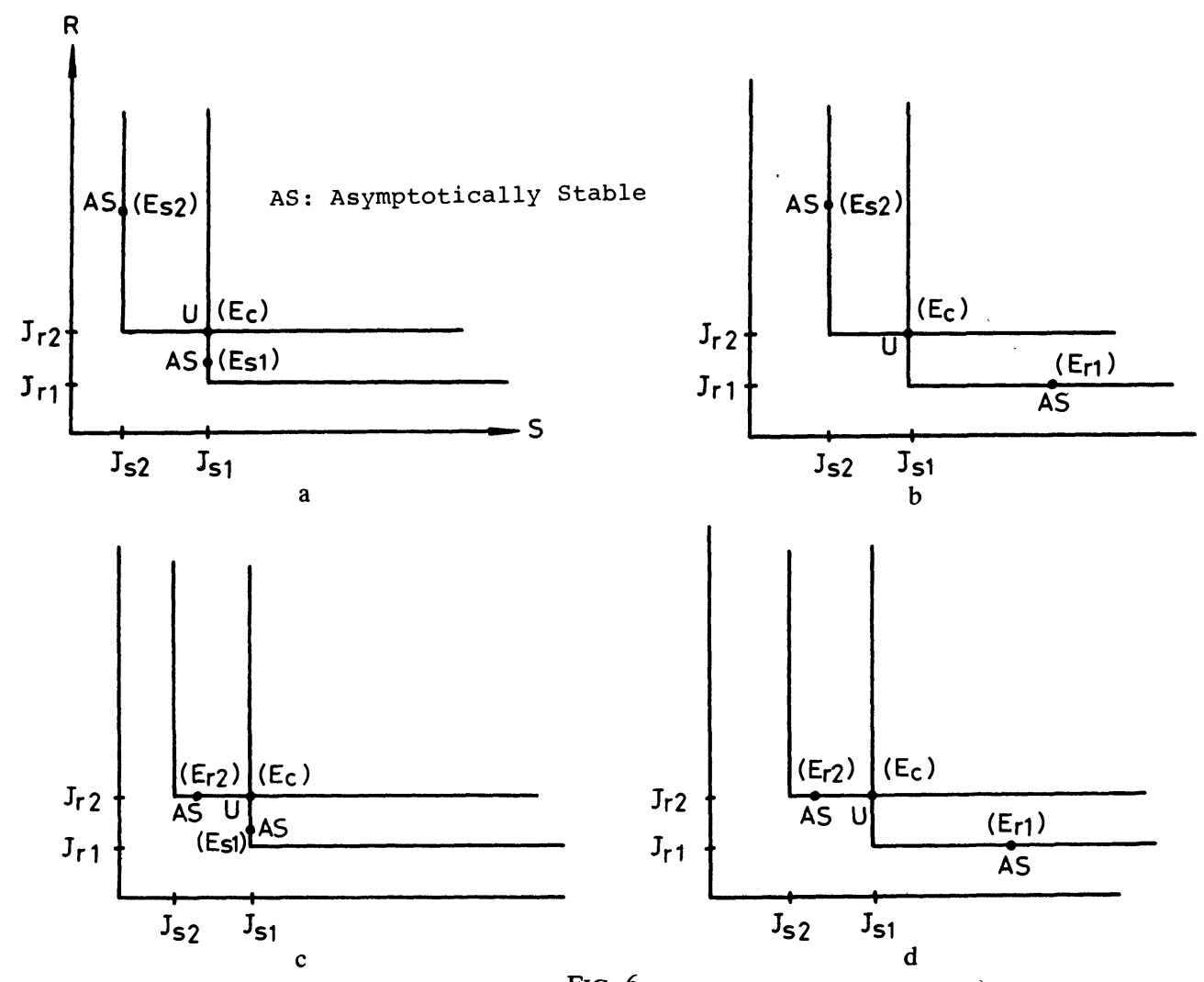

FIG. 6 
(b) If $N_{1}$ is $R$-limited and $N_{2}$ is $S$-limited, then $\left(E_{r 1}\right)$ and $\left(E_{s 2}\right)$ are asymptotically stable (see Fig. 6b).

(c) If $N_{1}$ is $S$-limited and $N_{2}$ is $R$-limited, then $\left(E_{s 1}\right)$ and $\left(E_{r 2}\right)$ are asymptotically stable (see Fig. 6c).

(d) If $N_{1}$ is $R$-limited and $N_{2}$ is $R$-limited, then $\left(E_{r 1}\right)$ and $\left(E_{r 2}\right)$ are asymptotically stable (see Fig. 6d).

This case arises because each species has the lower subsistence concentration for that resource which, at the two-species equilibrium mixture of resources, least limits the growth of its rival. This makes the two-species equilibrium unstable. The outcomes depend on whether species $N_{1}$ and species $N_{2}$ are individually $S$-limited or $R$-limited at the one-species equilibrium.

6. The proofs.

Proof of Lemma 5.2. From (4.1), it follows that

$$
N_{i}(t)=N_{i}(0) \exp \left(\int_{0}^{t}\left[\min \left(\frac{m_{s i} S(\xi)}{K_{s i}+S(\xi)}, \frac{m_{r i} R(\xi)}{K_{r i}+R(\xi)}\right)-D\right] d \xi\right) .
$$

Let $\varepsilon>0$ be chosen such that

$$
\min \left(\frac{m_{s i}\left(S^{(0)}+\varepsilon\right)}{K_{s i}+\left(S^{(0)}+\varepsilon\right)}-D, \frac{m_{r i}\left(R^{(0)}+\varepsilon\right)}{K_{r i}+\left(R^{(0)}+\varepsilon\right)}-D\right)<0,
$$

and from (5.1), (5.2) choose $t_{0}>0$ such that $S(t) \leqq S^{(0)}+\varepsilon, R(t) \leqq R^{(0)}+\varepsilon, t \geqq t_{0}$. Then, for an appropriate constant $C$, it follows that

$$
N_{i}(t) \leqq C N_{i}(0) \exp \left\{\left[\min \left(\frac{m_{s i}\left(S^{(0)}+\varepsilon\right)}{K_{s i}+\left(S^{(0)}+\varepsilon\right)}-D, \frac{m_{r i}\left(R^{(0)}+\varepsilon\right)}{K_{r i}+\left(R^{(0)}+\varepsilon\right)}-D\right)\right] \cdot\left(t-t_{0}\right)\right\}
$$

Hence $\lim _{t \rightarrow \infty} N_{i}(t)=0$. Q.E.D.

Before we prove Theorem 5.4(i), (ii), (iii), (iv), Theorem 5.5 and Theorem 5.6, we present the following idea which reduces the problem of the four-dimensional system of differential equations (4.1) to a problem of a two-dimensional system of differential equations.

Consider the Lyapunov function

$$
V\left(S, R, N_{1}, N_{2}\right)=\left(S+\sum_{i=1}^{2} \frac{N_{i}}{y_{s i}}-S^{(0)}\right)^{2}+\left(R+\sum_{i=1}^{2} \frac{N_{1}}{y_{r i}}-R^{(0)}\right)^{2}
$$

for (4.1). It follows that

$$
\dot{V}=-2\left[\left(S+\sum_{i=1}^{2} \frac{N_{i}}{y_{s i}}-S^{(0)}\right)^{2}+\left(R+\sum_{i=1}^{2} \frac{N_{i}}{y_{r i}}-R^{(0)}\right)^{2}\right] \leqq 0 .
$$

Hence

$$
\begin{aligned}
& E=\left\{\left(S, R, N_{1}, N_{2}\right): \dot{V}=0\right\}=\left\{\left(S, R, N_{1}, N_{2}\right): S^{(0)}=S+\sum_{i=1}^{2} \frac{N_{i}}{y_{s i}},\right. \\
&\left.R^{(0)}=R+\sum_{i=1}^{2} \frac{N_{i}}{y_{r i}}, S \geqq 0, R \geqq 0, N_{i} \geqq 0, i=1,2\right\} .
\end{aligned}
$$


Then the $\omega$-limit set $\Omega$ of the trajectory of (4.1) lies in $E$ [16], and it is sufficient to study the behavior of solutions of the following two-dimensional system:

$$
\begin{array}{cc}
\frac{d N_{1}}{d t}=N_{1} G_{1}\left(N_{1}, N_{2}\right), & \frac{d N_{2}}{d t}=N_{2} G_{2}\left(N_{1}, N_{2}\right), \\
N_{1}(0) \geqq 0, & N_{2}(0) \geqq 0,
\end{array}
$$

where

$$
G_{i}\left(N_{1}, N_{2}\right)=g_{i}\left(S^{(0)}-\sum_{i=1}^{2} \frac{N_{i}}{y_{s i}}, R^{(0)}-\sum_{i=1}^{2} \frac{N_{i}}{y_{r i}}\right)-D, \quad i=1,2 .
$$

Proof of Theorem 5.4. First we prove (i) and (ii). Since (5.3) holds for $i=2$, from Lemma 5.2 we have $\lim _{t \rightarrow \infty} N_{2}(t)=0$. Then the trajectory of (4.1) approaches $E \cap\left\{\left(S, R, N_{1}, N_{2}\right): N_{2}=0\right\}$, and it suffices to consider the equation $d N_{1} / d t=$ $N_{1} G_{1}\left(N_{1}, 0\right), N_{1}(0) \geqq 0$, or, equivalently,

$$
\begin{gathered}
\frac{d N_{1}}{d t}=N_{1}\left[\min \left\{\frac{\left(m_{s 1}-D\right)\left(S^{(0)}-J_{s 1}-N_{1} / y_{s 1}\right)}{K_{s 1}+S^{(0)}-N_{1} / y_{s 1}}, \frac{\left(m_{r 1}-D\right)\left(R^{(0)}-J_{r 1}-N_{1} / y_{r 1}\right)}{K_{r 1}+R^{(0)}-N_{1} / y_{r 1}}\right\}\right], \\
N_{1}(0) \geqq 0 .
\end{gathered}
$$

If $T_{1}>C_{1}$, i.e., $\left(R^{(0)}-J_{r 1}\right) y_{r 1}>\left(S^{(0)}-J_{s 1}\right) y_{s 1}$, then $N_{1}=0$ and $N_{1}=\left(S^{(0)}-J_{s 1}\right) y_{s 1}$ are the only two possible equilibria of (6.2). And $\lim _{t \rightarrow \infty} N_{1}(t)=N_{s 1}^{*}=y_{s 1} \cdot\left(S^{(0)}-J_{s 1}\right)$, provided $N_{1}(0)>0$ in $(6.2)$. Since $0<J_{s 1}<S^{(0)}, 0<J_{r 1}<R^{(0)}$, from the third equation of (4.1) it is impossible that $\lim _{t \rightarrow \infty} N_{1}(t)=0$. Then there exists $\left(\bar{S}, \bar{R}, \bar{N}_{1}, 0\right) \in \Omega$ with $\bar{N}_{1}>0$. Then part (i) follows directly from the invariance property of $\omega$-limit set and the fact $\left(E_{s 1}\right)$ is asymptotically stable.

If $T_{1}<C_{1}$, then using the above arguments yields the proof of (ii). The proof for part (iii), (iv) respectively is similar to that of part (i) and (ii). Q.E.D.

Before we prove Theorem 5.5 and Theorem 5.6, we state a theorem of Markus [40] which will be used repeatedly.

Definition. Let $A: x_{i}^{\prime}=f_{i}(x, t)$ and $A_{\infty}: x_{i}^{\prime}=f_{i}(x)(i=1.2, \cdots, n)$ be a first-order system of ordinary differential equations. The real-valued functions $f_{i}(x, t)$ and $f_{i}(x)$ for continuous in $(x, t)$ for $x \in G$, where $G$ is an open subset of $R^{n}$, and for $t>t_{0}$ : they satisfy a local Lipschitz condition in $x$. $A$ is said to be asymptotic to $A_{\infty}\left(A \rightarrow A_{\infty}\right)$ in $G$ if, for each compact set $K \subseteq G$ and for each $\varepsilon>0$, there is a $T=T(K, \varepsilon)>t_{0}$ such that $\left|f_{i}(x, t)-f_{i}(x)\right|<\varepsilon$ for all $i=1,2, \cdots, n$, all $x \in K$, all $t>T$.

DEFINITION. The $\Omega$-limit set for $x^{\prime}=f(x, t), x\left(t_{0}\right)=x_{0}$ is the set of $\omega$-limit points $y$, where $y=\lim _{n \rightarrow \infty} x\left(t_{n}\right)$ for some sequence $\left\{t_{n}\right\}, t_{n} \rightarrow \infty$.

THEOREM (Markus). Let $A \rightarrow A_{\infty}$ in $G$ and let $P$ be an asymptotically stable critical point of $A_{\infty}$. Then there is a neighborhood $N$ of $P$ and time $T$ such that the $\Omega$ limit set for every solution $x(t)$ of $A$ which intersects $N$ at a time later than $T$ is equal to $P$.

Next, we need to describe the isoclines $d N_{1} / d t=0, d N_{2} / d t=0$ of (6.1) for various cases. The proofs will be based on these geometric figures. First we note that the transformation

$$
S=S^{(0)}-\sum_{i=1}^{2} \frac{N_{i}}{y_{s i}}, \quad R=R^{(0)}-\sum_{i=1}^{2} \frac{N_{i}}{y_{r i}}
$$

is $1-1$ from the $S-R$ plane into the $N_{1}-N_{2}$ plane provided $C_{1} \neq C_{2}$. The equations in 
(6.1) can be rewritten as

$$
\begin{aligned}
& \frac{d N_{1}}{d t}=N_{1}\left[\min \left\{\frac{\left(m_{s 1}-D\right)\left(S^{(0)}-J_{s 1}-\frac{N_{1}}{y_{s 1}}-\frac{N_{2}}{y_{s 2}}\right)}{K_{s 1}+S^{(0)}-\frac{N_{1}}{y_{s 1}}-\frac{N_{2}}{y_{s 2}}}, \frac{\left(m_{r 2}-D\right)\left(R^{(0)}-J_{r 1}-\frac{N_{1}}{y_{r 1}}-\frac{N_{2}}{y_{r 2}}\right)}{K_{r 1}+R^{(0)}-\frac{N_{1}}{y_{r 1}}-\frac{N_{2}}{y_{r 2}}}\right\},\right. \\
& \frac{d N_{2}}{d t}=N_{2}\left[\min \left\{\frac{\left(m_{s 2}-D\right)\left(S^{(0)}-J_{s 2}-\frac{N_{1}}{y_{s 1}}-\frac{N_{2}}{y_{s 2}}\right)}{K_{s 2}+S^{(0)}-\frac{N_{1}}{y_{s 1}}-\frac{N_{2}}{y_{s 2}}}, \frac{\left(m_{r 1}-D\right)\left(R^{(0)}-J_{r 2}-\frac{N_{1}}{y_{r 1}}-\frac{N_{2}}{y_{r 2}}\right)}{K_{r 2}+R^{(0)}-\frac{N_{1}}{y_{r 1}}-\frac{N_{2}}{y_{r 2}}}\right\}\right], \\
& N_{1}(0) \geqq 0, \quad N_{2}(0) \geqq 0
\end{aligned}
$$

The isocline $d N_{i} / d t=0, i=1,2$, in the $N_{1}-N_{2}$ plane can be classified into four cases by mapping the isocline in the $S-R$ plane (see Fig. 1) under the transformation (6.3).

Case 1. $T_{i} \geqq C_{1}, C_{2}, i=1,2$ (see Fig. 7a).

Case 2. $T_{i} \leqq C_{1}, C_{2}, i=1,2$ (see Fig. 7b).

Case 3. $C_{1} \leqq T_{i} \leqq C_{2}, i=1,2$ (see Fig. 7c).

Case 4. $C_{2} \leqq T_{i} \leqq C_{1}, i=1,2$ (see Fig. 7d).

We note that the isoclines of (6.1) or (6.4) are similar to those in the Lotka-Volterra competition model (1.1), and hence we can study the behavior of solutions of (6.1) by isocline analysis or by pushing trajectory.

Proof of Theorem 5.5. Assume $T_{1}>C_{1}$. Since $C_{1} \neq C_{2}$, the nonsingular transformation (6.3) maps two disjoint isoclines of (4.1) in Fig. $2 \mathrm{a}$ or $2 \mathrm{~b}$ into two disjoint isoclines of (6.1) in the $N_{1}-N_{2}$ plane.

Combining the two isoclines $d N_{1} / d t=0, d N_{2} / d t=0$ of (6.1) yields seven various figures. The isocline $d N_{1} / d t=0$ of (6.2) is of the type in Fig. 7a or Fig. 7c. On the other hand, the isocline $d N_{2} / d t=0$ has four various forms. These forms are similar in our discussion, and we only need to pick one of them in this proof. For example, Fig. 8 is the combination of Fig. 7a, Fig. 7d respectively for isoclines $d N_{1} / d t=0, d N_{2} / d t=0$

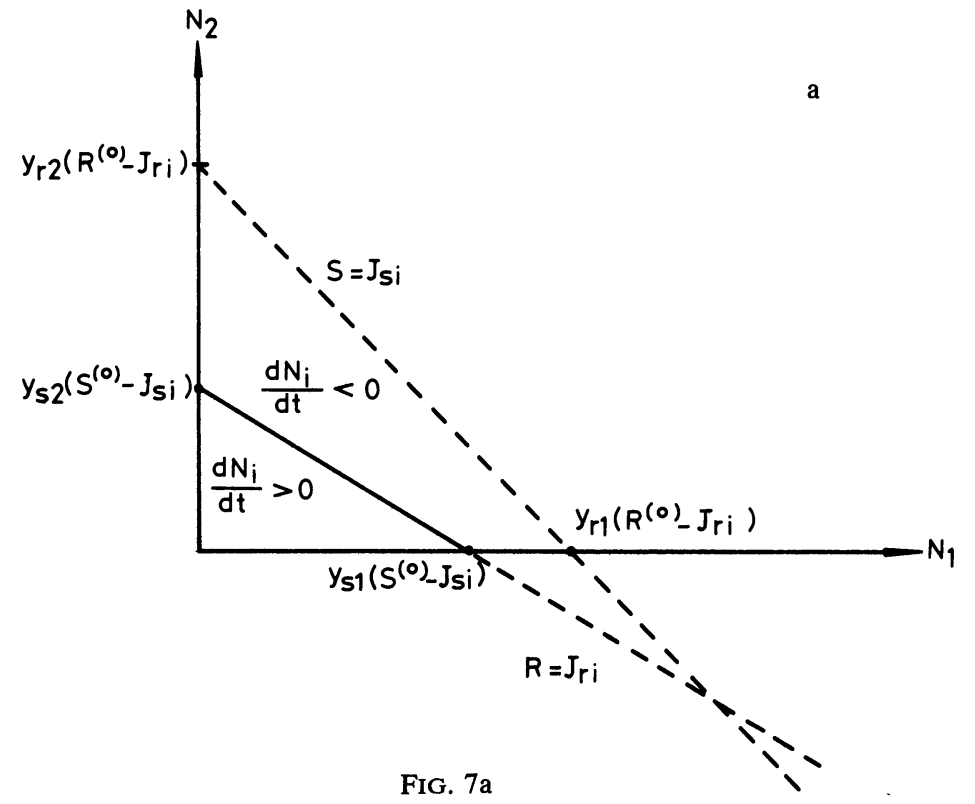



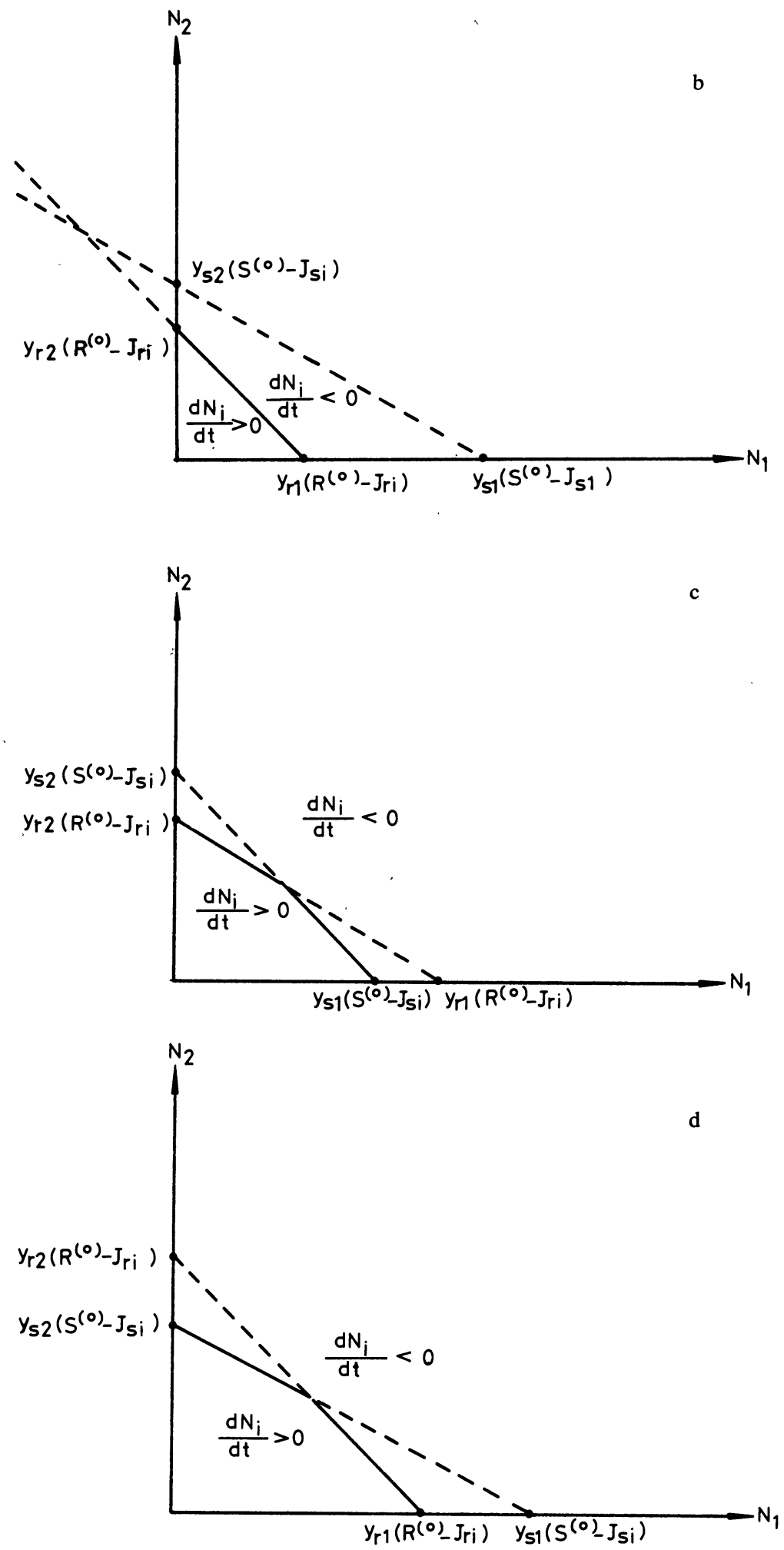

FIG. 7b-d 


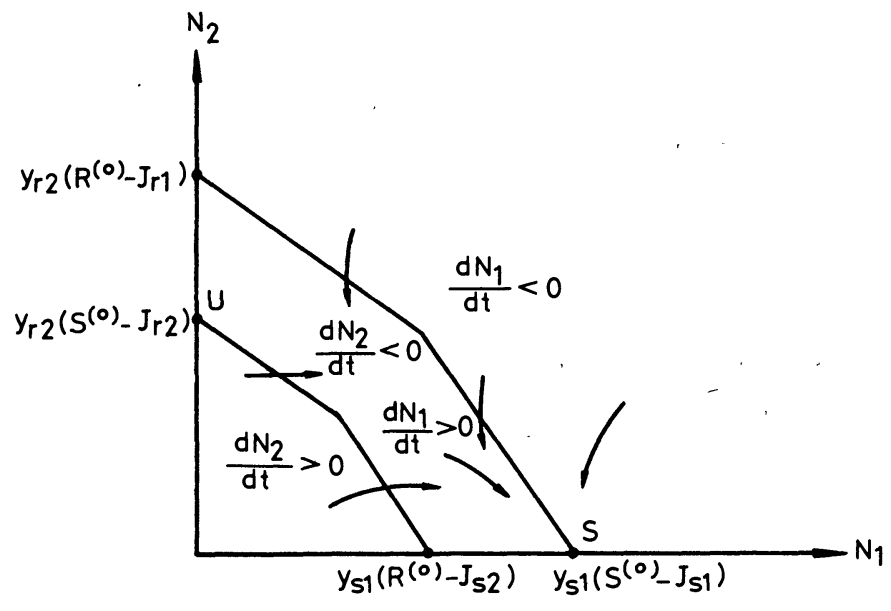

FIG. 8

(see Fig. 8). If $N_{1}(0)>0, N_{2}(0) \geqq 0$ then, from isocline analysis, the trajectory of (6.2) approaches $\left(y_{s 1}\left(S^{(0)}-J_{s 1}\right), 0\right)$.

Consider the trajectory $\left(S(t), R(t), N_{1}(t), N_{2}(t)\right)$ of (4.1). First we claim $\lim _{t \rightarrow \infty} N_{1}(t) \neq 0$. Suppose $\lim _{t \rightarrow \infty} N_{1}(t)=0$; then from (H1) and Lemma 5.1 we have $\lim _{t \rightarrow \infty} N_{2}(t) \neq 0$. Hence there exists a point $\left(\bar{S}, \bar{R}, 0, \bar{N}_{2}\right) \in \Omega$ for some $\bar{S} \geqq 0, \bar{R}>0$, $\bar{N}_{2}>0$ where $\Omega$ is the $\omega$-limit set of the trajectory $\left(S(t), R(t), N_{1}(t), N_{2}(t)\right)$. By the invariance property of the $\omega$-limit set, we have $\left(E_{s 2}\right) \in \Omega$ (if $T_{2}>C_{2}$ ) or $\left(E_{r 2}\right) \in \Omega$ (if $T_{2}<C_{2}$ ). Compare the following two systems of differential equations:

$$
\begin{gathered}
\frac{d S}{d t}=\left(S^{(0)}-S\right) D-\frac{1}{y_{s 1}} g_{1}(S, R) N_{1}-\frac{1}{y s_{2}} g_{2}(S, R) N_{2} \\
\frac{d R}{d t}=\left(R^{(0)}-R\right) D-\frac{1}{y_{r 1}} g_{1}(S, R) N_{1}-\frac{1}{y_{r 2}} g_{2}(S, R) N_{2}, \\
\frac{d N_{2}}{d t}=\left[g_{2}(S, R)-D\right] N_{2} ; \\
\frac{d S}{d t}=\left(S^{(0)}-S\right) D-\frac{1}{y_{s 2}} g_{2}(S, R) N_{2}, \\
\frac{d R}{d t}=\left(R^{(0)}-R\right) D-\frac{1}{y_{r 2}} g_{2}(S, R) N_{2}, \\
\frac{d N_{2}}{d t}=\left[g_{2}(S, R)-D\right] N_{2} .
\end{gathered}
$$

Obviously, under the assumption $\lim _{t \rightarrow \infty} N_{1}(t)=0$ we have that (6.5) is asymptotic to (6.6). Since $\left(E_{s 2}\right) \in \Omega$ (if $\left.T_{2}>C_{2}\right)$ or $\left(E_{r 2}\right) \in \Omega$ (if $\left.T_{2}<C_{2}\right)$ and $\left(\hat{E}_{r 2}\right)$ or $\left(\hat{E}_{s 2}\right)$ is asymptotically stable for system (6.6), where $\left(\hat{E}_{r 2}\right)=\left(S_{r 2}^{*}, J_{r 2}, N_{r 2}^{*}\right),\left(\hat{E}_{s 2}\right)=\left(J_{s 2}, R_{s 2}^{*}, N_{s 2}^{*}\right)$, Markus' theorem yields that $\lim _{t \rightarrow \infty} S(t)=S_{r 2}^{*}, \lim _{t \rightarrow \infty} R(t)=J_{r 2}, \lim _{t \rightarrow \infty} N_{2}(t)=$ $N_{r 2}^{*}$ or $\lim _{t \rightarrow \infty} S(t)=J_{s 2}, \lim _{t \rightarrow \infty} R(t)=R_{s 2}^{*}, \lim _{t \rightarrow \infty} N_{2}(t)=N_{s 2}^{*}$. Either case implies the unboundedness of $N_{1}(t)$. (see Fig. 2a, Fig. 2b). This is the desired contradiction. Hence $\lim _{t \rightarrow \infty} N_{1}(t) \neq 0$. 
Since $\lim _{t \rightarrow \infty} N_{1}(t) \neq 0$, it follows that there exists a point $\left(\hat{S}, \hat{R}, \hat{N}_{1}, \hat{N}_{2}\right) \in \Omega$ with $\hat{S} \geqq 0, \hat{R} \geqq 0, \hat{N}_{1}>0, \hat{N}_{2} \geqq 0$. The trajectory $\left(N_{1}(t), N_{2}(t)\right)$ with initial values $N_{1}(0)=$ $\hat{N}_{1}, N_{2}(0)=\hat{N}_{2}$ approaches $\left(N_{s 1}^{*}, 0\right)$. This and the invariance property of the $\omega$-limit set, $\Omega \in E$, imply $\left(E_{s 1}\right) \in \Omega$. But $\left(E_{s 1}\right)$ is asymptotically stable. Hence $\lim _{t \rightarrow \infty}(S(t), R(t)$, $\left.N_{1}(t), N_{2}(t)\right)=\left(E_{s 1}\right)$.

For the case $T_{1}<C_{2}$, similar arguments yield that $\left(E_{r 1}\right)$ is globally asymptotically stable. Q.E.D.

Proof of Theorem 5.6(i), (ii). First we prove part (i). The proof of part (ii) is similar to that of part (i) and we omit it. Since $J_{r 1}<J_{r 2}, J_{s 2}<J_{s 1}$, it follows that

$$
T^{*}=\frac{R^{(0)}-J_{r 2}}{S^{(0)}-J_{s 1}}<T_{1}=\frac{R^{(0)}-J_{r 1}}{S^{(0)}-J_{s 1}}, \quad T^{*}=\frac{R^{(0)}-J_{r 2}}{S^{(0)}-J_{s 1}}>T_{2}=\frac{R^{(0)}-J_{r 2}}{S^{(0)}-J_{s 2}} .
$$

From the assurnption $T^{*}<C_{1}, C_{2}$, we have $T_{2}<C_{1}, C_{2}$. Applying the assumptions (H3) and $T^{*}<C_{1}, C_{2}$ yields four possible cases for the forms of the isoclines $d N_{1} / d t=0, d N_{2} / d t=0$ of $(6.2)$.

Case 1. $T_{1} \geqq C_{1}, C_{2}$ (see Fig. 9a which corresponds to Fig. 3a).

Case 2. $T_{1} \leqq C_{1}, C_{2}$ (see Fig. $9 \mathrm{~b}$ which corresponds to Fig. $3 \mathrm{~b}$ ).

Case 3. $C_{1} \leqq T_{1} \leqq C_{2}$ (see Fig. 9c which corresponds to Fig. 3a).

Case 4. $C_{2} \leqq T_{1} \leqq C_{1}$ (see Fig. 9d which corresponds to Fig. 3b). Using the argument of Theorem 5.5 yields the proof of part (i). Q.E.D.

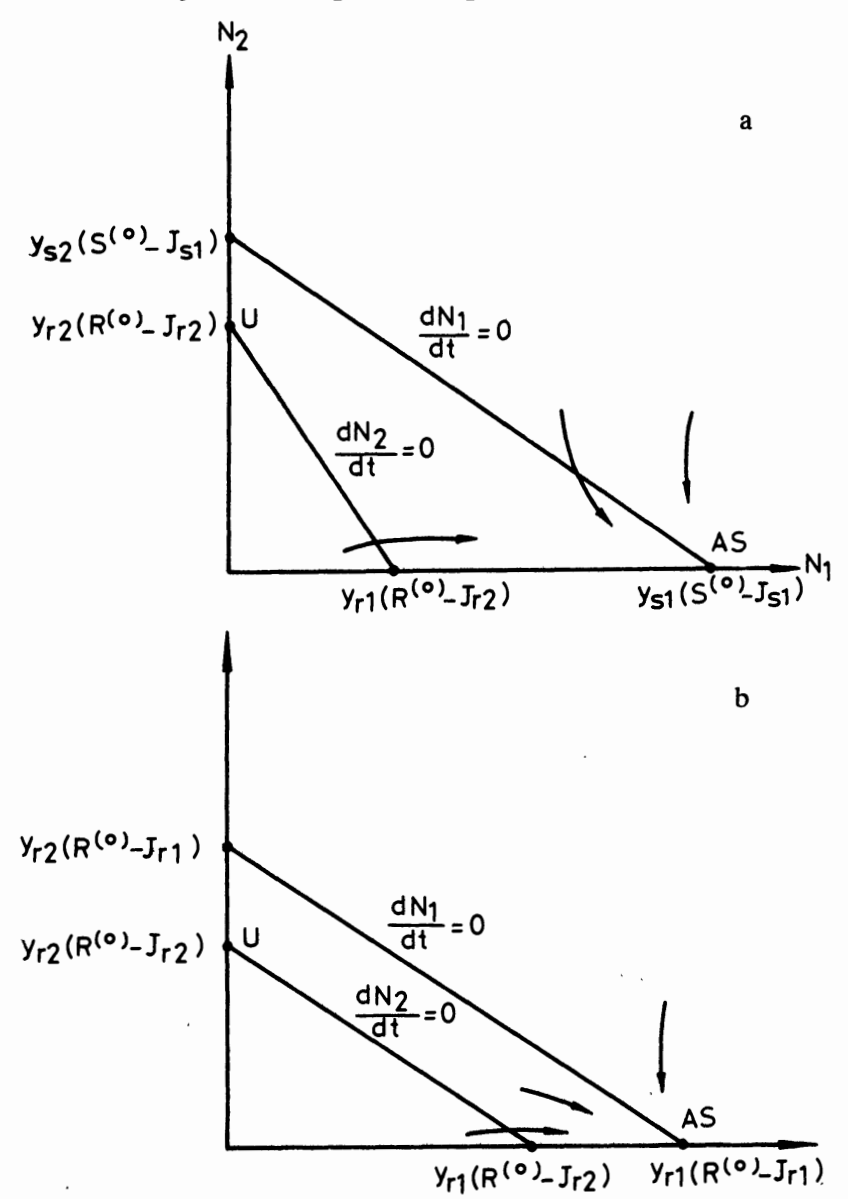

FIG. 9a-b 

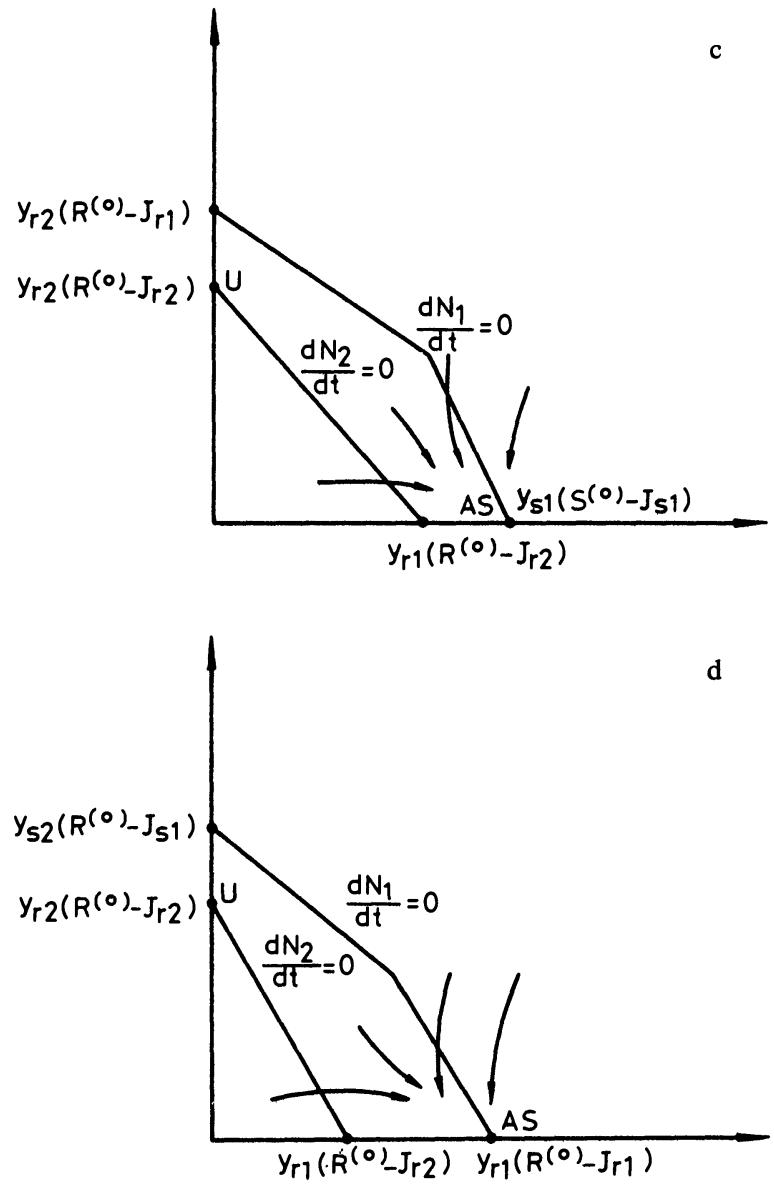

FIG. 9c-d

Proof of Theorem 5.6 (iii). We note that under assumption (H3) we have $T^{*}<$ $T_{1}, T^{*}>T_{2}$. Since $C_{1}<T^{*}<C_{2}$ we have four possible cases which all corespond to Fig. 5:

Case 1. $C_{1} \leqq T_{1} \leqq C_{2}, C_{1} \leqq T_{2} \leqq C_{2}$ (see Fig. 10a).

Case 2. $C_{1} \leqq T_{2} \leqq C_{2}, T_{1} \geqq C_{1}, C_{2}$ (see Fig. 10b).

Case 3. $C_{1} \leqq T_{1} \leqq C_{2}, T_{2} \leqq C_{1}, C_{2}$ (see Fig. 10c).

Case 4. $T_{2} \leqq C_{1}, C_{2}, T_{1} \geqq C_{1}, C_{2}$ (see Fig. 10d).

First we note that from linear stability analysis $\left(E_{c}\right)$ is asymptotically stable. Using the similar arguments in the proof of Theorem 5.5 yields that $\lim _{t \rightarrow \infty} N_{1}(t) \neq 0$, $\lim _{t \rightarrow \infty} N_{2}(t) \neq 0$. By the same arguments as in the proof of Theorem 5.5 it suffices to show that there exists a point $\left(\bar{S}, \bar{R}, \bar{N}_{1}, \bar{N}_{2}\right) \in \Omega$ with $\bar{N}>0, \bar{N}_{2}>0$. We have the following three possible cases.

Case a. $N_{1}(t) \geqq N_{1 c}^{*}$ for all $t \geqq t_{0}$ for some $t_{0}$. Since $\lim _{t \rightarrow \infty} N_{2}(t) \neq 0$, there exists a point $\left(\bar{S}, \bar{R}, \bar{N}_{1}, \bar{N}_{2}\right) \in \Omega$ with $\bar{N}_{1} \geqq N_{1 c}^{*}, \bar{N}_{2} \geqq \varepsilon$ for some $\varepsilon>0$.

Case b. $N_{1}(t) \leqq N_{1 c}^{*}$ for all $t \geqq t_{0}$ for some $t_{0}$. In this case we have two subcases:

Subcase $1 . \lim _{t \rightarrow \infty} N_{1}(t)=c>0$ for some $c$. Since $\lim _{t \rightarrow \infty} N_{2}(t) \neq 0$, there exists a point $\left(\bar{S}, \bar{R}, c, \bar{N}_{2}\right) \in \Omega$ with $\bar{N}_{1}=c>0, \bar{N}_{2} \geqq \varepsilon$ for some $\varepsilon>0$.

Subcase $2 . \lim _{t \rightarrow \infty} N_{1}(t)$ does not exist. Then there exist $\varepsilon>0$ and a sequence $\left\{t_{n}\right\}$ with $N_{1}\left(t_{n}\right)>\varepsilon$ and $\left(d N_{1} / d t\right)\left(t_{n}\right)=0$. We may choose a subsequence $\left\{t_{n i}\right\}$ such that 
440

S.-B. HSU, K.-S. CHENG, AND S. P. HUBBELL
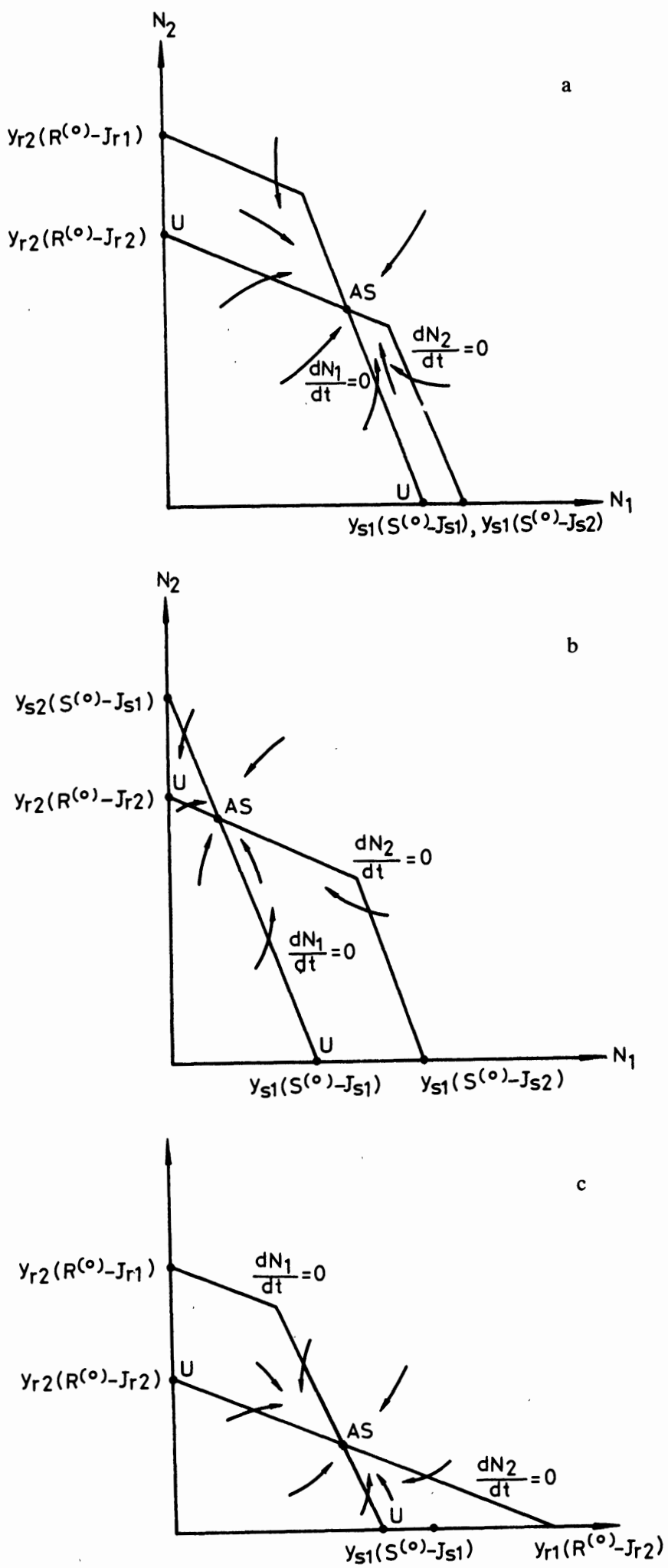

FIG. 10a-c 


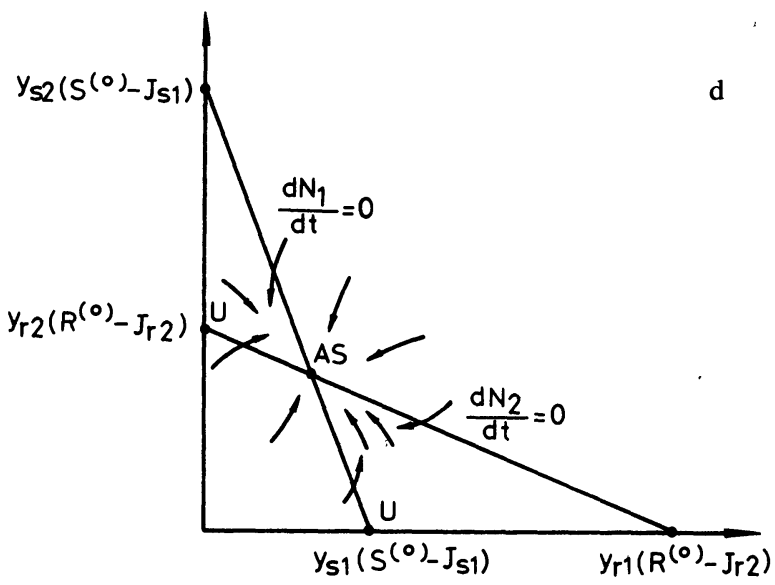

FIG. 10d

either $S\left(t_{n i}\right)=J_{s 1}$ for all $t_{n i}$ or $R\left(t_{n i}\right)=J_{r 1}$ for all $t_{n i}$. If $S\left(t_{n i}\right)=J_{s 1}$ for all $t_{n i}$, then from (5.1) we have

$$
\begin{aligned}
N_{2}\left(t_{n i}\right) & =y_{s 2}\left[\left(S^{(0)}-J_{s 1}\right)-\frac{N_{1}\left(t_{n i}\right)}{y_{s 1}}\right]+o(1) \\
& \geqq y_{s 2}\left[\left(S^{(0)}-J_{s 1}\right)-\frac{N_{1 c}^{*}}{y_{s 1}}\right]+o(1)=N_{2 c}^{*}+o(1) .
\end{aligned}
$$

Let $t_{n i} \rightarrow \infty$ and choose an appropriate subsequence of $t_{n i}$; there exists a point $\left(J_{s 1}, \bar{R}\right.$, $\left.\bar{N}_{1}, \bar{N}_{2}\right) \in \Omega$ with $\bar{N}_{1} \geqq \varepsilon$ and $\bar{N}_{2} \geqq N_{2 c}^{*}$. If $R\left(t_{n i}^{\prime}\right)=J_{r 1}$ for all $t_{n i}$, then from (5.2) we have

$$
\begin{aligned}
N_{2}\left(t_{n i}\right) & =y_{r 2}\left[\left(R^{(0)}-J_{r 1}\right)-\frac{N_{1}\left(t_{n i}\right)}{y_{r 1}}\right]+o(1) \\
& \geqq y_{r 2}\left[\left(R^{(0)}-J_{r 2}\right)-\frac{N_{1 c}^{*}}{y_{r 1}}\right]+o(1)=N_{2 c}^{*}+O(1) .
\end{aligned}
$$

Hence there exists a point $\left(\bar{S}, J_{r 1}, \bar{N}_{1}, \bar{N}_{2}\right) \in \Omega$ with $\bar{N}_{1} \geqq \varepsilon$ and $\bar{N}_{2} \geqq N_{2 c}^{*}$.

Case c. $N_{1}(t)$ oscillates around $N_{1}=N_{1 c}^{*}$. Then there exists $\left\{t_{n}\right\}$ with $\left(d N_{1} / d t\right)\left(t_{n}\right)<0$ and $N_{1}\left(t_{n}\right)=N_{1 c}^{*}$. In this case we may choose a subsequence $\left\{t_{n i}\right\}$ such that either $S\left(t_{n i}\right) \leqq J_{s 1}$ for all $t_{n i}$ or $R\left(t_{n i}\right) \leqq J_{r 1}$ for all $t_{n i}$. Then from (5.1) or (5.2) we still have inequalities (6.7) and (6.8). Hence we complete the proof of Theorem 5.6(iii). Q.E.D.

Proof of Theorem 5.6(iv). From the assumption that $C_{2}<T^{*}<C_{1}$, the "positive" equilibrium $\left(E_{c}\right)$ exists. That $\left(E_{c}\right)$ is unstable follows directly from the assumption $C_{2}<T^{*}<C_{1}$, and the linear stability analysis about $\left(E_{c}\right)$. The results are obvious from Figs. 6a, 6b, 6c, 6d. Q.E.D.

Remark. In describing Theorems 5.5 and 5.6 we take $C_{1} \neq C_{2}$ as an essential assumption. What can happen when $C_{1}=C_{2}$ ? The meaning for $C_{1}=C_{2}$ is that the fixed yield ratio for species 1 growing on resources $S$ and $R$ is equal to the fixed yield ratio for species 2 growing on resources $S$ and $R$. In this case, the isoclines in (6.4) are parallel lines in the $N_{1}-N_{2}$ plane. Since the proofs are the same as or even more simple than the proofs for the case $C_{1} \neq C_{2}$, we merely state the results here and omit the proofs. 
Let us define for convenience

$$
N_{i j}=\min \left\{y_{s j}\left(S^{(0)}-J_{s i}\right), y_{r j}\left(R^{(0)}-J_{r i}\right)\right\}, \quad i, j=1,2,
$$

and without loss of generality assume that $J_{s 1}<J_{s 2}$.

(i) If $J_{r 1}<J_{r 2}$, then $N_{11}>N_{21}$ and

$$
\lim _{t \rightarrow+\infty}\left(S(t), R(t), N_{1}(t), N_{2}(t)\right)=\left(S^{(0)}-\frac{N_{11}}{y_{s 1}}, R^{(0)}-\frac{N_{11}}{y_{r 1}}, N_{11}, 0\right) .
$$

(ii) If $J_{r 2}<J_{r 1}$ and $N_{11}>N_{21}$, then

$$
\lim _{t \rightarrow+\infty}\left(S(t), R(t), N_{1}(t), N_{2}(t)\right)=\left(S^{(0)}-\frac{N_{11}}{y_{s 1}}, R^{(0)}-\frac{N_{11}}{y_{r 1}}, N_{11}, 0\right) .
$$

(iii) If $J_{r 2}<J_{r 1}$ and $N_{11}<N_{21}$, then

$$
\lim _{t \rightarrow+\infty}\left(S,(t), R(t), N_{1}(t), N_{2}(t)\right)=\left(S^{(0)}-\frac{N_{22}}{y_{s 2}}, R^{(0)}-\frac{N_{22}}{y_{r 2}}, 0, N_{22}\right) .
$$

(iv) If $J_{r 2}<J_{r 1}$ and $N_{11}=N_{21}$, then

$$
\lim _{t \rightarrow+\infty}\left(S(t), R(t), N_{1}(t), N_{2}(t)\right)=\left(J_{s 2}, J_{r 1}, N_{1}^{*}, N_{2}^{*}\right),
$$

which depends on initial conditions. $N_{1}^{*}$ and $N_{2}^{*}$ satisfy the following equation

$$
\frac{N_{1}^{*}}{y_{s 1}}+\frac{N_{2}^{*}}{y_{s 2}}=S^{(0)}-J_{s 2} \quad \text { or } \quad \frac{N_{1}^{*}}{y_{r 1}}+\frac{N_{2}^{*}}{y_{r 2}}=R^{(0)}-J_{r 1} \text {. }
$$

This means that all points on the line $N_{1} / y_{s 1}+N_{2} / y_{s 2}=S^{(0)}-J_{s 2}$ on the $N_{1}-N_{2}$ plane (on the nonnegative octant) are equilibrium points.

7. Discussion. In this paper, we have explored the behavior of an exploitative competition model which describes how two species compete for two complementary resources. The analysis has revealed that each of the classical outcomes of two-species Lotka-Volterra competition theory can arise in two or three different ways when resource dynamics and consumer-resource interaction are explicitly considered.

Leon and Tumpson [17] discuss the competition between two species for two complementary or substitutable resources. Interested readers may find the mathematical analysis and biological discussion for substitutable resources in a paper of Waltman, Hubbell and Hsu [37].

The equations in (4.1) describes how two species compete for two complementary nutrients in the chemostat. Tilman and Kilham [32] and Tilman [33] have performed interesting competition studies in semicontinuous cultures between two freshwater diatoms, Asterionella formosa Hass., and Cyclotella meneghiniana Kutz for the complementary resources phosphate and silicate. They did not report any cases in which the outcomes were dependent on initial numbers. However, they did find a broad region of coexistence over a range of ratios of silicate/phosphate in the influent supply to semicontinuous cultures of the two diatom species.

We have taken the data provided in Tilman [33] to see if there is any possibility of a case in which the initial number of Asterionella or Cyclotella would determine the outcome of competition. Let $J_{\mathrm{PA}}$ amd $J_{\mathrm{SA}}$ be the $J$ criteria for Asterionella on phosphate and silicate, respectively, and let $J_{\mathrm{PC}}$ and $J_{\mathrm{SC}}$ be the corresponding $J$ criteria for Cyclotella. If we assume that all cell death was due to washout from the culture in the effluent, then the maximum death rate they studied experimentally was $0.5 /$ day, i.e., 
$D=0.5 /$ day. Then the values of the $J$ criteria are $J_{\mathrm{PA}}=0.025 \mu \mathrm{M}$ (micromole), $J_{\mathrm{SA}}=$ $3.28 \mu \mathrm{M}, J_{\mathrm{PC}}=0.417 \mu \mathrm{M}$ and $J_{\mathrm{SC}}=0.90 \mu \mathrm{M}$. Thus $J_{\mathrm{PA}}<J_{\mathrm{PC}}$, so that Asterionella has a lower subsistence concentration on phosphate than Cyclotella by more than an order of magnitude, but $J_{\mathrm{CS}}<J_{\mathrm{As}}$, so that Cyclotella has a lower subsistence concentration on silicate than Asterionella.

Next, it is necessary to compute $T^{*}, \mathrm{C}_{\mathrm{A}}$, and $C_{\mathrm{C}}$, where $C_{\mathrm{A}}$ and $C_{\mathrm{C}}$ are the $C$ criteria for Asterionella and Cyclotella, respectively,

$$
T^{*}=\frac{\left(P^{(0)}-J_{\mathrm{PC}}\right)}{\left(S^{(0)}-J_{\mathrm{SA}}\right)},
$$

where $P^{(0)}$ and $S^{(0)}$ are the input phosphate and silicate concentrations, respectively, and the point $\left(J_{\mathrm{SA}}, J_{\mathrm{PC}}\right)$ is the intersection of the Asterionella and Cyclotella isoclines on the silicate-phosphate resource plane. Of the range of values of $P^{(0)}$ and $S^{(0)}$ tested by Tilman, we chose $P^{(0)}=10 \mu \mathrm{M}$. and $S^{(0)}=100 \mu \mathrm{M}$. This gives a value for $T^{*}=$ $9.9 \times 10^{-2}$.

Finally, it is necessary to compute the $C$ criteria for the two diatoms. The yield constants for Asterionella are reported by Tilman [33] to be: $Y_{\mathrm{PA}}=$ $2.18 \times 10^{8}$ cells $/ \mu \mathrm{M}$ on phosphate, and $Y_{\mathrm{SA}}=2.51 \times 10^{6}$ cells $/ \mu \mathrm{M}$ on silicate. Therefore, $C_{\mathrm{A}}=\left(1 / y_{\mathrm{PA}}\right) /\left(1 / y_{\mathrm{SA}}\right)=1.15 \times 10^{-2}$. The yield constants for Cyclotella are $Y_{\mathrm{PC}}=$ $2.59 \times 10^{7}$ cells $/ \mu \mathrm{M}$ on phosphate, $Y_{\mathrm{SC}}=4.20 \times 10^{6}$ cells $/ \mu \mathrm{M}$ on silicate. Thus, $C_{\mathrm{C}}=\left(1 / Y_{\mathrm{PC}}\right) /\left(1 / Y_{\mathrm{SC}}\right)=1.62 \times 10^{-1}$.

With this information, we can answer the question of whether there can exist a case in which the winning diatom species (Asterionella or Cyclotella) is determined by the initial cell density of each diatom. We note that $J_{\mathrm{PA}}<J_{\mathrm{PC}}$ and $J_{\mathrm{SA}}>J_{\mathrm{SA}}$. Next, we note that $C_{\mathrm{A}}<T^{*}<C_{\mathrm{C}}$. This corresponds to a case of coexistence, Theorem. 5.6(iii), a fact that Tilman [33] confirmed experimentally. In order for there to be a case in which the initial diatom density determines the outcome in this competitive system for these $J$ 's, it would be necessary that the inequalities among $C_{\mathrm{A}}, T^{*}$, and $C_{\mathrm{C}}$ be totally reversed: $C_{\mathrm{A}}>T^{*}>C_{\mathrm{C}}$. This, in turn, would require substantial changes in the yield constants for phosphate and silicate in these two diatom species. Since only the criterion variable $T^{*}$ involves parameters under experimental control, there is no possiblity of a case in which initial cell densities affect the competitive outcome between Asterionella and Cyclotella. We note that it is possible for $T^{*}<C_{\mathrm{A}}, C_{\mathrm{C}}$ or $T^{*}>C_{\mathrm{A}}, C_{\mathrm{C}}$ such that $T^{*}$ is an experimental parameter. In either of these cases, only one species survives and coexistence does not result.

\section{REFERENCES}

[1] M. R. DROOP, The nutrient status of algal cells in continuous culture, J. Marine Biol. Assoc. U.K., 54 (1974), pp. 825-855.

[2] R. C. DUGDALE, Nutrient limitation in sea dynamics: identification and significance, Limnol. Oceanogr. 12 (1974), pp. 685-695.

[3] R. W. EPPLEY AND J. L. COATSWORTH, Uptake of nitrate and nitrite by Pitylum brightwellii-Kinetics and mechanisms, J. Phycol., 4 (1968), pp. 151-158.

[4] R. W. EPPLEY AND W. H. THOMAS, Comparison of half-saturation constants for growth and nitrate uptake of marine phytoplankton, J. Phycol., 5 (1969), pp. 375-379.

[5] S. R. HANSEN AND S. P. HUBBELL, Single-nutrient microbial competition: agreement between experimental and theoretically forecast outcomes (subm. to Science, 1979).

[6] D. Herbert, R. Elsworth AND R. C. TElliNG, The continuous culture of bacteria: a theoretical and experimental study, J. Gen. Microbiol., 14 (1956), pp. 601-622.

[7] C. S. HolliNG, The components of predation as revealed by a study of small mammal predation of the European pine sawfly, Canad. Entomol., 91 (1959), pp. 293-320. 
[8] - The functional response of predators to prey density and its role in mimicry and population regulation, Mem. Entomol. Soc. Canada, 45 (1965), pp. 3-60.

[9] - The functional response of invertebrate predators to prey density, Mem. Entomol. Soc. Canada, 48 (1966), pp. 1-86.

[10] S.-B. Hsu, S. P. Hubbell AND P. WAltman, A mathematical theory of single-nutrient competition in continuous cultures of microorganisms, this Journal, 32 (1977), pp. 366-383.

[11] S.-B. HsU, Limiting behavior for competing species, this Journal, 34 (1978), pp. 760-763.

[12] S.-B. Hsu, S. P. Hubbell AND P. WAltman, A contribution to the theory of competing predators, Ecological Monographs, 48 (1978), pp. 337-349.

[13] S.-B. Hsu, A competition model for a seasonally fluctuating environment, J. Math. Biology, to appear.

[14] P. KILHAM, A hypothesis concerning silica and the freshwater planktonic diatoms, Limnol. Oceanogr., 16 (1971), pp. 10-18.

[15] A. L. КосH, Competitive coexistence of two predators utilizing the same prey under constant environmental conditions, J. Theoret. Biol., 44 (1974), pp. 373-386.

[16] J. P. LASAlle, The Stability of Dynamical Systems, CBMS Regional Conference Series in Applied Mathematics 25, Society for Industrial and Applied Mathematics, Philadelphia, 1976.

[17] J. A. LEON, AND D. B. Tumpson, Competition between two species for two complementary or two substitutable resources, J. Theoret. Biol., 50 (1975), pp. 185-201.

[18] A. J. LotKA, Elements of Mathematical Biology, Williams and Wilkins, Baltimore, MD, 1925.

[19] R. H. MACARTHUR, Geographical Ecology, Harper and Row, New York, 1972.

[20] R. MCGehee AND R. A. ARMSTRONG, Some mathematical problems concerning the ecological principle of competitive exclusion, J. Differential Equations, 23 (1977), pp. 30-52.

[21] R. S. Miller, Pattern and process in competition, Adv. Ecol. Res., 4 (1976), pp. 1-47.

[22] W. E. NeILl, The community matrix and interdependence of the competition coefficients, Amer. Nat., 108 (1974), 399-408.

[23] R. H. PeTers, Tautology in evolution and ecology, Amer. Nat., 110 (1976), pp. 1-12.

[24] E. O. POWELL, Criteria for growth of contaminants and mutants in continuous culture, J. Gen. Microbiol. 18 (1958), pp. 259-268.

[25] L. A. REAL, The kinetics of functional response, Amer. Nat., 111 (1977), pp. 287-300.

[26] R. C. Richmond, M. E. Gilpin, S. Perez Salas and E. J. Ayala, A search for emergent competitive phenomena: the dynamics of multispecies Drosophila systems, Ecology, 56 (1975), pp. 709-714.

[27] M. L. RosENZWEIG, Evolution of the predator isocline, Evolution, 27 (1973), pp. 84-94.

[28] T. W. SCHOENER, Some methods for calculating competitive coefficients from resource utilization spectra, Amer. Nat., 108 (1974), pp. 320-340.

[29] O. Smith, H. H. Shugart, R. V. O'Neill, R. S. Booth and D. C. McNaught, Resource competition and an analytical model of zooplankton feeding on phytoplankton, Amer. Nat., 109 (1975), pp. 571-591.

[30] F. M. STEWART, AND B. R. LeVIN, Partitioning of resources and the outcome of interspecific competition: a model and some general considerations, Amer. Nat., 107 (1973), pp. 171-198.

[31] P. A. TAYLOR, AND J. L. WILlIAMS, Theoretical studies on the coexistence of competing species under continuous-flow conditions, Canad. J. Microbiol. 21 (1975), pp. 90-98.

[32] D. Tilman, AND S. S. Kilham, Phosphate and silicate growth and uptake kinetics of the diatoms, Asterionella formosa and Cyclotella meneghiniana, J. Phycol., 12 (1976), pp. 375-383.

[33] D. TILMAN, Resource competition between planktonic algae: an experimental and theoretical approach, Ecology, 58 (1977), pp. 338-348.

[34] J. H. VANDERMEER, The competitive structure of communities: an experimental approach with protozoa, Ecology, 50 (1969), pp. 361-371.

[35] P. H. VERHULST, Notice sur la loi que la population pursuit dans son accroissement, Correspond. Math. Phys., 10 (1838), pp. 113-121.

[36] V. VOLTERRA, Variations and fluctuations of the number of individuals of animal species living together, Animal Ecology, R. N. Chapman, ed., McGraw-Hill, New York, 1926.

[37] P. E. Waltman, S. P. Hubbell ANd S.-B. Hsu, Theoretical and experimental investigation of microbial competition in continuous cultures, Proc. of Conf. on Mathematical Modelling, Carbondale, Il., 1979.

[38] P. J. WANGERSKY, Lotka-Volterra population models. Ann. Rev. Ecol. Syst., 9 (1978), pp. 189-218.

[39] H. WILBER, Competition, predation, and the structure of the Ambystoma-Rana Sylvatica community, Ecology 53 (1972), pp. 3-21.

[40] L. MARKuS, Asymptotically autonomous differential systems, in Contributions to the Theory of Nonlinear Oscillation, vol. 3, Princeton University Press, Princeton, N.J., 1956, pp. 17-29. 